Document downloaded from:

http://hdl.handle.net/10251/156432

This paper must be cited as:

Gregori Verdú, S.; Tur Valiente, M.; Pedrosa Sanchez, AM.; Tarancón Caro, JE.; Fuenmayor Fernández, F. (2019). A modal coordinate catenary model for the real-time simulations of the pantograph-catenary dynamic interaction. Finite Elements in Analysis and Design. 162:1-12. https://doi.org/10.1016/j.finel.2019.05.001

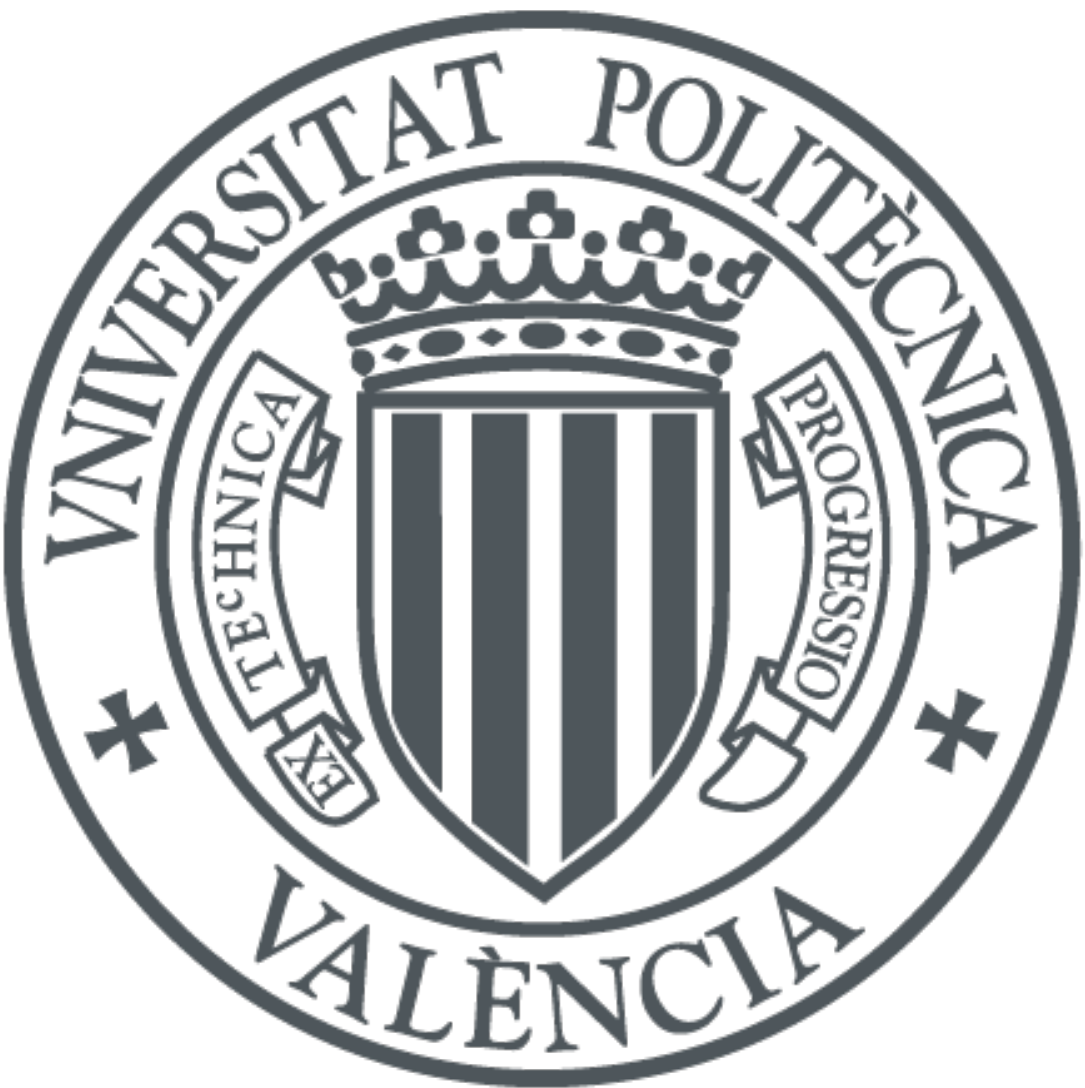

The final publication is available at

https://doi.org/10.1016/j.finel.2019.05.001

Copyright Elsevier

Additional Information 


\title{
A modal coordinate catenary model for the real-time simulation of the pantograph-catenary dynamic interaction
}

\author{
S. Gregori ${ }^{* 1}$, M. Tur ${ }^{\dagger 1}$, A. Pedrosa ${ }^{\ddagger 1}$, J. E. Tarancón ${ }^{\S 1}$, and F. J. \\ Fuenmayor $₫ 1$
}

${ }^{1}$ Centro de Investigación en Ingeniería Mecánica, Universitat Politècnica de València, Camino de Vera s/n, 46022 Valencia, Spain

May 20, 2019

\begin{abstract}
The computational cost required to simulate the pantograph-catenary dynamic interaction can be a limiting factor in certain applications. Specifically, for Hardwarein-the-Loop (HIL) simulations, real-time capabilities of the software are imperative. In this paper a combination of a modal coordinate approach with an offline/online strategy to build a very efficient simulation strategy is proposed. This novel approach preserves the accuracy of the results, compared with those obtained by classical finite element strategies. Furthermore, a procedure to define and validate a criterion for a priori truncation of the modal basis and an analysis of the effect of explicit treatment of the interaction force are also presented. The results show that the method proposed could be used to perform pantograph HIL tests.
\end{abstract}

*Corresponding author. E-mail: sangreve@upv.es; Tel. +34 963877621 ORCID ID: 0000-0002-0483-3531

†E-mail: manuel.tur@mcm.upv.es; ORCID ID: 0000-0001-7683-4771

${ }^{\ddagger}$ E-mail: anpedsan@dimm.upv.es; ORCID ID: 0000-0001-8069-5620

§E-mail: jetaranc@mcm.upv.es; ORCID ID: 0000-0002-1469-5304

๑E-mail: ffuenmay@mcm.upv.es; ORCID ID: 0000-0003-3594-9593 


\section{Introduction}

The overhead contact line, commonly known as the catenary, is the system in charge of supplying power to the locomotive by means of a sliding interaction with the pantograph, which is located on the roof of the locomotive. The coupled pantograph-catenary dynamic behaviour can be a limiting factor when intending to increase the service speed [1]. Thus, the design of such systems [2] is aimed to provide the most uniform interaction force as possible to prevent both wearing of the contact surfaces and arcing, with the consequent damage and power supply deterioration.

Today, the numerical simulation of the pantograph-catenary dynamic interaction is a well-established technique, as evidenced by the existence of specific standards [3] and the completion of the benchmark exercise [4]. These simulation tools can be used to speed up the catenary design process since the effect on the dynamic behaviour of varying geometrical and material parameters can be efficiently evaluated. Usually, the models used in the simulation codes assume certain simplifications of the real systems. Regarding the pantograph, a linear lumped mass model is commonly used [4], in which the nonlinear effects of friction, bump stops, actuating system, etc... [5], and the flexibility of the pantograph elements $[6,7]$, are rarely considered. In any case, they are taken into account with uncertainty in the model parameters.

Another issue related to the pantograph is the large number of field tests required to fulfil regulations for its homologation to be used in a given locomotive. These tests usually need a long time and high investment to be carried out. In this sense, Hardware-in-theLoop (HIL) simulation is revealed to be a promising tool to palliate both the uncertainty in the pantograph modelling and the large number of expensive in-line tests [8]. These hybrid simulations consist of the interaction of a real pantograph device with a simulated catenary numerical model.

During the last decade, some authors have proposed different strategies to deal with this problem. The first research into this field can be found in [9], in which the catenary displacement is expressed as a sum of sine terms and the dropper slackening is not considered. Later, the group of Professor Bruni presented several publications regarding HIL pantograph tests [10-14]. They include realistic effects such as dropper slackening and 
conoloretact wire stagger, and proposed a 'shift-forward' procedure to run the simulations beyond the limited section of the catenary modelled. More recently, Schirrer et al. $[15,16]$, presented a high-speed pantograph test rig to perform HIL tests with a novel catenary representation in moving coordinates. In this case, they propose the use of absorbing boundary conditions to allow the use of a limited computational catenary domain.

The goal of this paper is to improve the catenary models that can be used for HIL tests, i.e. to provide a simulation strategy able to simulate the dynamic response of a whole catenary section in real-time, while keeping the accuracy of the results compared with those obtained in the standard finite element simulations. Although the proposed approach is based on that presented in [17], in this work, the catenary coordinates are projected to a modal basis which can be properly truncated. The modal truncation along with an efficient dynamic integration strategy provide the model with the necessary features to be a candidate in an HIL set-up.

The paper is organised as follows. The real-time model is presented in Section 2, with special emphasis on the modal coordinate transformation and all the calculations performed at each stage of the method. The adaptation of the algorithm to explicitly deal with the interaction force, is described and validated in Section 3. As accuracy is the main pillar of the method proposed in this paper, Section 4 is fully devoted to expose different modal basis truncation techniques and their repercussions in the accuracy of the results. Then, an analysis of the computational time required by the proposed simulation method is conducted in Section 5. Finally, some concluding remarks are provided in Section 6.

\section{The real-time simulation method}

The proposed algorithm is based on the efficient Offline/Online simulation strategy presented in [17], but some changes that improve the computational cost by a factor of 5 are introduced here. In particular, the degrees of freedom (DOF) of the catenary model are projected to a modal basis and a new parameter is defined to truncate this modal basis without compromising the accuracy of the simulations.

The following sections are devoted to thoroughly presenting the calculations performed during each of the steps of the simulation method presented. 


\subsection{The pantograph-catenary dynamic interaction problem}

Regarding the numerical models of the different subsystems that appear in this problem, the Finite Element Method (FEM) is used to model the catenary. Specifically, the Absolute Nodal Coordinate Formulation (ANCF) proposed in [18] is adopted in this work.

The pantograph is modelled by a lumped-parameter model, which only introduces vertical DOF, whereas the interaction between the pantograph and the catenary models is accomplished by using the penalty method. A scheme of such a model is provided in Figure 1. The interested reader is referred to [17] for a detailed description of the formulation of these mathematical models.

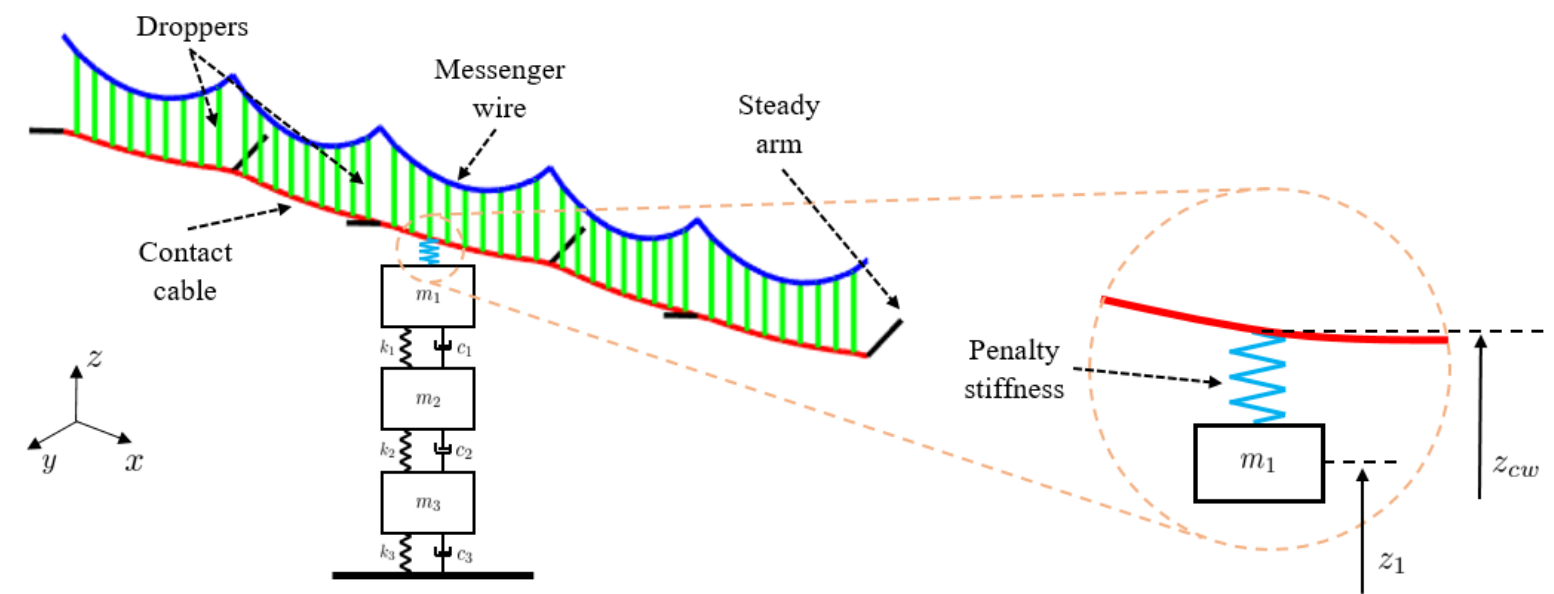

Figure 1: Catenary, pantograph and interaction models.

When designing a catenary, some targets, such as the height of the points at which the contact wire is connected to the droppers or the initial tension of the messenger and contact wires, are set for the catenary subjected to the gravity load. Thus, before addressing the pantograph-catenary interaction problem, the initial configuration of the catenary must be obtained. Among the different options in the literature to set and solve this so-called 'shape-finding' problem, the procedure proposed in [19] is used in this work, in which the nodal coordinates and the element lengths, fulfilling both the equilibrium equations and the aforementioned constraints imposed during catenary stringing, are obtained after solving a non-linear problem.

Due to the fact that the pantograph-catenary interaction dynamics is governed by small 
displacements, dynamic equations can be linearised with respect to the initial configuration, leading to the following semi-discrete system of differential equations:

$$
\mathbf{M} \ddot{\mathbf{u}}^{t}+\mathbf{C}^{t} \dot{\mathbf{u}}^{t}+\overline{\mathbf{K}}^{t} \mathbf{u}^{t}=\mathbf{F}^{t}
$$

which models the coupled behaviour of the whole system. The nodal DOF of displacement are denoted by $\mathbf{u}^{t}$, and their first and second time derivatives by $\dot{\mathbf{u}}^{t}$ and $\ddot{\mathbf{u}}^{t}$ respectively. $\mathbf{M}$ is the constant mass matrix, $\overline{\mathbf{K}}^{t}$ is the stiffness matrix and $\mathbf{F}^{t}$ is the external force vector. The catenary damping is considered in the matrix $\mathbf{C}^{t}$ with a proportional Rayleigh model. The superscript $t$ denotes the time dependence henceforth.

Despite the linearisation process followed to obtain Eq. (1), there are two severe nonlinearities in the system that must be taken into account, namely pantograph-catenary interaction including possible contact loss and dropper slackening. They cause the stiffness matrix (and therefore the damping matrix due to the Rayleigh assumption) as well as the external force vector to depend on time.

The first unilateral behaviour of the system is produced by the sliding contact between the pantograph and the contact wire and the possibility of having pantograph detachments. If $k_{h}$ is the penalty stiffness and $z_{1}^{t}$ and $z_{c w}^{t}$ are the vertical absolute coordinates of the top mass of the pantograph and the contact point on the contact wire (see Figure 1), respectively, the non-linear interaction force is:

$$
f_{\text {int }}^{t}= \begin{cases}k_{h}\left(z_{1}^{t}-z_{c w}^{t}\right) & \text { if } \quad z_{1}^{t}>z_{c w}^{t} \\ 0 & \text { if } \quad z_{1}^{t} \leq z_{c w}^{t}\end{cases}
$$

The vertical absolute coordinates are defined as the sum of the vertical position for the configuration at which the problem is linearised (in this case the initial configuration) and the vertical displacement with respect to this configuration. Specifically, for the top mass of the pantograph model and the interaction point on the contact wire, this means:

$$
\begin{aligned}
& z_{1}^{t}=z_{1}^{0}+u_{1}^{t} \\
& z_{c w}^{t}=z_{c w, 0}^{t}+u_{c w}^{t}
\end{aligned}
$$

where $z_{c w, 0}^{t}$ depends on time because the contact point on the contact wire evolves in time while the pantograph moves forward and $z_{1}^{0}$ has been chosen to match with $z_{c w, 0}^{0}$, 
i.e. the vertical position of the catenary interaction point when $t=0$. With the previous definitions, the interaction force becomes:

$$
f_{\text {int }}^{t}= \begin{cases}k_{h}\left(z_{1}^{0}-z_{c w, 0}^{t}\right)+k_{h}\left(u_{1}^{t}-u_{c w}^{t}\right)=f_{\text {int }, 0}^{t}+k_{h}\left(u_{1}^{t}-u_{c w}^{t}\right) & \text { if } z_{1}^{t}>z_{c w}^{t} \\ 0 & \text { if } z_{1}^{t} \leq z_{c w}^{t}\end{cases}
$$

When the two terms making up the interaction force are assembled to the global size problem (1), they contribute to the external force vector and the global stiffness matrix with $\mathbf{F}_{\text {int } 0}^{t}$ and $\mathbf{K}_{\text {int }}^{t}$ respectively, so that

$$
\overline{\mathbf{K}}^{t}=\mathbf{K}^{t}+\mathbf{K}_{\text {int }}^{t} \quad \mathbf{F}^{t}=\mathbf{F}_{\text {ext }}+\mathbf{F}_{\text {int }}^{t}
$$

in which $\mathbf{K}^{t}$ considers the catenary and the pantograph stiffness and $\mathbf{F}_{\text {ext }}$ represents the constant force applied to the pantograph by its uplift mechanism. The construction of all these matrices and vectors are detailed in [17].

The second non-linearity comes from droppers which, like any cable, are not able to undergo compressive forces. Given that Eq. (1) has been obtained from linearising with respect to the initial configuration position, as proposed in [20], the non-linear behaviour of droppers can be assimilated to the bilinear curve shown in Figure 2, where $f_{d j, 0}>0$ denotes the traction force of the dropper $j$ in the initial configuration position.

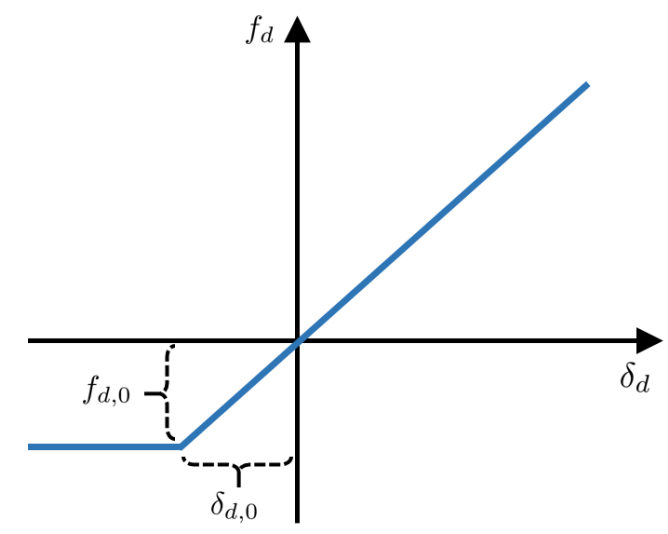

Figure 2: Bilinear force-elongation curve for droppers.

With this simplification, the force transmitted by the $j$-th dropper element, $f_{d j}^{t}$, can be 
obtained from its stiffness matrix $\mathbf{k}_{\mathbf{d} j}$ and the DOF vector $\mathbf{u}_{\mathbf{d} j}{ }_{j}$, is:

$$
f_{d j}^{t}=\left\{\begin{array}{ccc}
\left\langle\mathbf{k}_{\mathbf{d} j} \mathbf{u}_{\mathbf{d} j}^{t}\right\rangle & \text { if } \quad\left\langle\mathbf{k}_{\mathbf{d} j} \mathbf{u}_{\mathbf{d} j}^{t}\right\rangle>-f_{d j, 0} \\
-f_{d j, 0} & \text { if } & \left\langle\mathbf{k}_{\mathbf{d} j} \mathbf{u}_{\mathbf{d}_{j}}^{t}\right\rangle \leq-f_{d j, 0}
\end{array}\right.
$$

where the operator $\langle\cdot\rangle$ extracts the force of the lower node and projects it to the direction of the dropper.

If the Newmark method is used to solve Eq. (1), given the solution at time-step $t-1$, i.e. $\mathbf{u}^{t-1}, \dot{\mathbf{u}}^{t-1}$ and $\ddot{\mathbf{u}}^{t-1}$, the solution at time $t$ can be obtained through:

$$
\mathbf{K}_{I T}^{t} \mathbf{u}^{t}=\mathbf{F}_{I T}^{t}
$$

where

$$
\begin{aligned}
& \mathbf{K}_{I T}^{t}=\overline{\mathbf{K}}^{t}+b_{4} \mathbf{C}^{t}+b_{1} \mathbf{M} \\
& \mathbf{F}_{I T}^{t}=\mathbf{F}^{t}+\mathbf{M}\left(b_{1} \mathbf{u}^{t-1}+b_{2} \dot{\mathbf{u}}^{t-1}+b_{3} \ddot{\mathbf{u}}^{t-1}\right)+\mathbf{C}^{t}\left(b_{4} \mathbf{u}^{t-1}+b_{5} \dot{\mathbf{u}}^{t-1}+b_{6} \ddot{\mathbf{u}}^{t-1}\right)
\end{aligned}
$$

The coefficients $b_{i}, i=1, \ldots, 6$ are:

$$
\begin{array}{lll}
b_{1}=\frac{1}{\beta \Delta t^{2}} & b_{2}=-\frac{1}{\beta \Delta t} & b_{3}=1-\frac{1}{2 \beta} \\
b_{4}=\gamma \Delta t b_{1} & b_{5}=1+\gamma \Delta t b_{2} & b_{6}=\Delta t\left(1+\gamma b_{3}-\gamma\right)
\end{array}
$$

and depend on the time-step size $\Delta t$, and the Newmark method parameters $\beta=0.25$ and $\gamma=0.5$, which ensure unconditional stability and second-order accuracy for linear time-invariant systems.

Due to the aforementioned two non-linearities of the problem, Eq. (7) must be solved iteratively. In each time-step $t$, if $N_{s d}^{t, i}$ droppers are slackened in the current $i$-th iteration, the stiffness and damping matrices along with the external force vector present in Eq. (8) need to be modified as:

$$
\mathbf{K}_{i+1}^{t}=\mathbf{K}^{t}-\sum_{j}^{N_{s d}^{t, i}} \mathbf{K}_{\mathbf{d} j} \quad \mathbf{C}_{i+1}^{t}=\mathbf{C}^{t}-\sum_{j}^{N_{s d}^{t, i}} \mathbf{C}_{\mathbf{d} j} \quad \mathbf{F}_{i+1}^{t}=\mathbf{F}^{t}+\sum_{j}^{N_{s d}^{t, i}} \mathbf{F}_{\mathbf{d} j, 0}
$$

where $\mathbf{K}_{\mathbf{d} j}, \mathbf{C}_{\mathbf{d} j}$ and $\mathbf{F}_{\mathbf{d} j, 0}$ are the stiffness matrix, the damping matrix and the force 
vector in the initial configuration of the slackened dropper $j$, respectively, expanded to the problem global size.

Then, the contact state of the pantograph needs to be checked by applying Eq. (2). If contact loss occurs, $\mathbf{F}_{\text {int } 0}^{t}$ and $\mathbf{K}_{\text {int }}^{t}$ are set at null, and the iterative procedure follows to obtain $\mathbf{u}^{t}$ until two consecutive iterations provide with the same slackened droppers and interaction force. Finally, the velocities and accelerations are computed following the Newmark rules:

$$
\begin{aligned}
& \dot{\mathbf{u}}^{t}=b_{4}\left(\mathbf{u}^{t}-\mathbf{u}^{t-1}\right)+b_{5} \dot{\mathbf{u}}^{t-1}+b_{6} \ddot{\mathbf{u}}^{t-1} \\
& \ddot{\mathbf{u}}^{t}=b_{1}\left(\mathbf{u}^{t}-\mathbf{u}^{t-1}\right)+b_{2} \dot{\mathbf{u}}^{t-1}+b_{3} \ddot{\mathbf{u}}^{t-1}
\end{aligned}
$$

and the time integration process can move to the next time-step $t+1$.

Note that this direct procedure to solve the pantograph-catenary dynamic interaction problem deals with the non-linearities by solving iteratively the global size system of Eq. (7), in which the matrix $\mathbf{K}_{I T}^{t}$ needs to be modified in each iteration. This leads to a computationally intensive algorithm which cannot be solved in real-time.

The first action to alleviate computational cost, consists of moving the non-linear terms to the right hand side of Eq. (1) as proposed in [20]. This means moving the force related to the pantograph-catenary interaction and the correction force of the slackened droppers to the right hand side of Eq. (1), so that:

$$
\mathbf{M} \ddot{\mathbf{u}}^{t}+\mathbf{C} \dot{\mathbf{u}}^{t}+\mathbf{K} \mathbf{u}^{t}=\mathbf{F}^{t}+\mathbf{F}_{\text {int }}^{t}\left(\mathbf{u}^{t}\right)+\mathbf{F}_{\mathbf{d}}^{t}\left(\mathbf{u}^{t}\right)
$$

where

$$
\begin{aligned}
& \mathbf{F}_{\text {int }}^{t}\left(\mathbf{u}^{t}\right)=\mathbf{F}_{\text {int } 0}^{t}-\mathbf{K}_{\text {int }}^{t} \mathbf{u}^{t} \\
& \mathbf{F}_{\mathbf{d}}^{t}\left(\mathbf{u}^{t}\right)=\sum_{j}^{N_{s d}^{t}} \mathbf{F}_{\mathbf{d} j, 0}+\mathbf{K}_{\mathbf{d} j} \mathbf{u}^{t}+\mathbf{C}_{\mathbf{d} j} \dot{\mathbf{u}}^{t}
\end{aligned}
$$


Eq. (12) can be split into pantograph $\mathbf{u}_{p}^{t}$, and catenary $\mathbf{u}_{c}^{t}$, DOF as:

$$
\begin{aligned}
\left(\begin{array}{cc}
\mathbf{M}_{c} & \mathbf{0} \\
\mathbf{0} & \mathbf{M}_{p}
\end{array}\right)\left(\begin{array}{c}
\ddot{\mathbf{u}}_{c}^{t} \\
\ddot{\mathbf{u}}_{p}^{t}
\end{array}\right)+\left(\begin{array}{cc}
\mathbf{C}_{c} & \mathbf{0} \\
\mathbf{0} & \mathbf{C}_{p}
\end{array}\right)\left(\begin{array}{c}
\dot{\mathbf{u}}_{c}^{t} \\
\dot{\mathbf{u}}_{p}^{t}
\end{array}\right)+\left(\begin{array}{cc}
\mathbf{K}_{c} & \mathbf{0} \\
\mathbf{0} & \mathbf{K}_{p}
\end{array}\right)\left(\begin{array}{c}
\mathbf{u}_{c}^{t} \\
\mathbf{u}_{p}^{t}
\end{array}\right)= \\
\left(\begin{array}{c}
\mathbf{0} \\
\mathbf{F}_{\text {ext,p }}
\end{array}\right)+\left(\begin{array}{l}
\mathbf{F}_{\text {into,c }}^{t} \\
\mathbf{F}_{\text {into,p }}^{t}
\end{array}\right)-\left(\begin{array}{ll}
\mathbf{K}_{\text {int,cc }}^{t} & \mathbf{K}_{\text {int }, c p}^{t} \\
\mathbf{K}_{\text {int }, p c}^{t} & \mathbf{K}_{\text {int }, p p}
\end{array}\right)\left(\begin{array}{c}
\mathbf{u}_{c}^{t} \\
\mathbf{u}_{p}^{t}
\end{array}\right)+\left(\begin{array}{c}
\mathbf{F}_{\mathbf{d}, c}^{t} \\
\mathbf{0}
\end{array}\right)
\end{aligned}
$$

being $\mathbf{M}_{c}, \mathbf{C}_{c}$ and $\mathbf{K}_{c}$ the mass, damping and stiffness matrices of the catenary system, and $\mathbf{M}_{p}, \mathbf{C}_{p}$ and $\mathbf{K}_{p}$ for the pantograph.

As can be seen in Eq. (14), the correction force of the slackened droppers $\mathbf{F}_{\mathbf{d}, c}^{t}\left(\mathbf{u}_{c}^{t}\right)$, only depends on the catenary DOF and the only link between catenary and pantograph is present in the non-linear force due to interaction $\mathbf{F}_{\text {int }}^{t}\left(\mathbf{u}^{t}\right)$, which depends on both catenary and pantograph coordinates. Furthermore, all the three matrices of the left hand side are constant, and therefore when applying the Newmark integration scheme, the new matrix $\mathbf{K}_{I T}$ does not depend on time any more. Thereby, although in each time-step the resulting system of equations needs to be solved iteratively, the LU factorisation of the matrix $\mathbf{K}_{I T}$ can be done only once. Furthermore, by applying the reverse Cuthill-McKee ordering, the bandwidth of the $\mathrm{L}$ and $\mathrm{U}$ matrices is reduced and all the nonzero elements are concentrated near the diagonal. This factorisation reduces notably the computational cost of the simulations as pointed out in [21], since solving the system results now in performing a backward and forward substitution.

\subsection{Modal basis formulation}

In the search to reduce the computational cost of the simulations, the first ingredient used in this work consists of projecting the catenary nodal coordinates to a modal basis. This allows the matrix $\mathbf{K}_{I T}$ to be diagonal, which contributes to increase the computational efficiency of the simulations, and furthermore, the modal basis can be truncated, leading to a reduction in the number of DOF of the problem.

For a catenary model with $N$ DOF, by solving the generalised eigenproblem with the matrices $\mathbf{M}_{c}$ and $\mathbf{K}_{c}$, the eigenvectors $\boldsymbol{\Psi}_{n}$ and the natural frequencies $\omega_{n}$ of the catenary, 
for $n=1, \ldots, N$, can be obtained. Thus, the coordinate transformation results in:

$$
\left(\begin{array}{c}
\mathbf{u}_{c}^{t} \\
\mathbf{u}_{p}^{t}
\end{array}\right)=\left(\begin{array}{cc}
\mathbf{\Psi} & \mathbf{0} \\
\mathbf{0} & \mathbf{I}
\end{array}\right)\left(\begin{array}{c}
\mathbf{q}_{c}^{t} \\
\mathbf{u}_{p}^{t}
\end{array}\right) \quad \rightarrow \quad \mathbf{u}^{t}=\mathbf{\Phi} \mathbf{q}^{t}
$$

where $\boldsymbol{\Psi}$ is the modal transformation matrix for the catenary coordinates, $\mathbf{I}$ is the identity matrix and $\mathbf{q}_{c}^{t}$ denotes the vector of catenary modal coordinates. As the size of the model associated with the pantograph is small, their coordinates are kept in the physical space.

The coordinate transformation defined in (15) can be applied to Eq. (12), which becomes into:

$$
\mathbf{M} \ddot{\mathbf{q}}^{t}+\mathbf{C} \dot{\mathbf{q}}^{t}+\mathbf{K} \mathbf{q}^{t}=\mathbf{F}_{e x t}+\mathbf{F}_{i n t}^{t}\left(\mathbf{q}^{t}\right)+\mathbf{F}_{\mathbf{d}}^{t}\left(\mathbf{q}_{c}^{t}\right)
$$

where the modal mass, damping and stiffness matrices:

$$
\mathbf{M}=\boldsymbol{\Phi}^{T} \mathbf{M} \boldsymbol{\mathbf { \Phi }} \quad \mathbf{C}=\boldsymbol{\Phi}^{T} \mathbf{C} \boldsymbol{\mathbf { S }} \quad \mathbf{K}=\boldsymbol{\Phi}^{T} \mathbf{K} \boldsymbol{\Phi}
$$

have a diagonal block related to the catenary coordinates due to the orthogonality properties of the modal basis and the proportional damping model used. The modal force vectors are:

$$
\begin{aligned}
& \mathbf{F}_{e x t}=\boldsymbol{\Phi}^{T} \mathbf{F}_{e x t} \\
& \mathbf{F}_{i n t}^{t}\left(\mathbf{q}^{t}\right)=\mathbf{F}_{i n t 0}^{t}+\mathbf{K}_{i n t}^{t} \mathbf{q}^{t} \\
& \mathbf{F}_{\mathbf{d}}^{t}\left(\mathbf{q}_{c}^{t}\right)=\sum_{j}^{N_{s d}^{t}} \mathbf{F}_{\mathbf{d} j, 0}+\mathbf{K}_{\mathbf{d} j} \mathbf{q}_{c}^{t}+\mathbf{C}_{\mathbf{d} j} \dot{\mathbf{q}}_{c}^{t}
\end{aligned}
$$

where

$$
\begin{array}{lll}
\mathbf{F}_{i n t 0}^{t}=\boldsymbol{\Phi}^{T} \mathbf{F}_{i n t 0}^{t} & \mathbf{K}_{i n t}^{t}=\boldsymbol{\Phi}^{T} \mathbf{K}_{i n t}^{t} \boldsymbol{\Phi} & \mathbf{F}_{\mathbf{d} j, 0}=\boldsymbol{\Phi}^{T} \mathbf{F}_{\mathbf{d} j, 0} \\
\mathbf{K}_{\mathbf{d} j}=\boldsymbol{\Phi}^{T} \mathbf{K}_{\mathbf{d} j} \boldsymbol{\Phi} & \mathbf{C}_{\mathbf{d} j}=\boldsymbol{\Phi}^{T} \mathbf{C}_{\mathbf{d} j} \boldsymbol{\Phi} &
\end{array}
$$

In terms of catenary and pantograph DOF, Eq. (16) can be now written as:

$$
\begin{aligned}
&\left(\begin{array}{cc}
\mathbf{M}_{c} & \mathbf{0} \\
\mathbf{0} & \mathbf{M}_{p}
\end{array}\right)\left(\begin{array}{c}
\ddot{\mathbf{q}}_{c}^{t} \\
\ddot{\mathbf{u}}_{p}^{t}
\end{array}\right)+\left(\begin{array}{cc}
\mathbf{C}_{c} & \mathbf{0} \\
\mathbf{0} & \mathbf{C}_{p}
\end{array}\right)\left(\begin{array}{c}
\dot{\mathbf{q}}_{c}^{t} \\
\dot{\mathbf{u}}_{p}^{t}
\end{array}\right)+\left(\begin{array}{cc}
\mathbf{K}_{c} & \mathbf{0} \\
\mathbf{0} & \mathbf{K}_{p}
\end{array}\right)\left(\begin{array}{c}
\mathbf{q}_{c}^{t} \\
\mathbf{u}_{p}^{t}
\end{array}\right)= \\
&\left(\begin{array}{c}
\mathbf{0} \\
\mathbf{F}_{e x t, p}
\end{array}\right)+\left(\begin{array}{l}
\mathbf{F}_{i n t, c}^{t}\left(\mathbf{q}^{t}\right) \\
\mathbf{F}_{i n t, p}^{t}\left(\mathbf{q}^{t}\right)
\end{array}\right)+\left(\begin{array}{c}
\mathbf{F}_{\mathbf{d}, c}^{t}\left(\mathbf{q}_{c}^{t}\right) \\
\mathbf{0}
\end{array}\right)
\end{aligned}
$$

which can be solved in time by using the Newmark time integration method. 
Regarding the catenary system and following the procedure defined in Section 2.1, if the solution at time-step $t-1$ is given, $\mathbf{q}_{c}^{t}$ is obtained from:

$$
\mathbf{K}_{I T c} \mathbf{q}_{c}^{t}=\mathbf{F}_{I T c}^{t}
$$

where

$$
\begin{aligned}
& \mathbf{K}_{I T c}=\mathbf{K}_{c}+b_{4} \mathbf{C}_{c}+b_{1} \mathbf{M}_{c} \\
& \mathbf{F}_{I T c}^{t}=\mathbf{F}_{i n i, c}^{t}+\mathbf{F}_{i n t, c}^{t}\left(\mathbf{q}^{t}\right)+\mathbf{F}_{\mathbf{d}, c}^{t}\left(\mathbf{q}_{c}^{t}\right)
\end{aligned}
$$

and

$$
\mathbf{F}_{i n i, c}^{t}=\mathbf{M}_{c}\left(b_{1} \mathbf{q}_{c}^{t-1}+b_{2} \dot{\mathbf{q}}_{c}^{t-1}+b_{3} \ddot{\mathbf{q}}_{c}^{t-1}\right)+\mathbf{C}_{c}\left(b_{4} \mathbf{q}_{c}^{t-1}+b_{5} \dot{\mathbf{q}}_{c}^{t-1}+b_{6} \ddot{\mathbf{q}}_{c}^{t-1}\right)
$$

From Eqs. (21) and (22) is derived that $\mathbf{q}_{c}^{t}$ can be written as the response to three force terms, namely the force due to initial conditions $\mathbf{F}_{i n i, c}^{t}$, which is readily available since it depends on information of time-step $t-1$, the interaction force $\mathbf{F}_{i n t, c}^{t}\left(\mathbf{q}^{t}\right)$, which depends on both the catenary and the pantograph DOF, and the correction force of slackened droppers $\mathbf{F}_{\mathbf{d}, c}^{t}\left(\mathbf{q}_{c}^{t}\right)$, which only depends on the catenary modal coordinates. As the matrix $\mathbf{K}_{I T c}$ is constant, the superposition principle is applied leading to:

$$
\mathbf{q}_{c}^{t}=\mathbf{q}_{i n i, c}^{t}+\mathbf{q}_{i n t, c}^{t}+\sum_{j}^{N_{s d}^{t}} \mathbf{q}_{\mathbf{d} j, c}^{t}
$$

where the last two terms will be obtained as explained in Sections 2.3 and 2.4.

With regard to the pantograph dynamics, following the same strategy as for the catenary, the solution $\mathbf{u}_{p}^{t}$ can be obtained by solving the following system of equations:

$$
\mathbf{K}_{I T p} \mathbf{u}_{p}^{t}=\mathbf{F}_{I T p}^{t}
$$

in which

$$
\begin{aligned}
& \mathbf{K}_{I T p}=\mathbf{K}_{p}+b_{4} \mathbf{C}_{p}+b_{1} \mathbf{M}_{p} \\
& \mathbf{F}_{I T p}^{t}=\mathbf{F}_{i n i, p}^{t}+\mathbf{F}_{i n t, p}^{t}\left(\mathbf{q}^{t}\right)
\end{aligned}
$$

and

$$
\mathbf{F}_{i n i, p}^{t}=\mathbf{M}_{p}\left(b_{1} \mathbf{u}_{p}^{t-1}+b_{2} \dot{\mathbf{u}}_{p}^{t-1}+b_{3} \ddot{\mathbf{u}}_{p}^{t-1}\right)+\mathbf{C}_{p}\left(b_{4} \mathbf{u}_{p}^{t-1}+b_{5} \dot{\mathbf{u}}_{p}^{t-1}+b_{6} \ddot{\mathbf{u}}_{p}^{t-1}\right)+\mathbf{F}_{e x t, p}
$$

In this case, only two force terms appear, namely the known initial conditions force term 
$\mathbf{F}_{\text {ini,p }}^{t}$ and the interaction force contribution $\mathbf{F}_{\text {int }, p}^{t}\left(\mathbf{q}^{t}\right)$, which again depends on both the catenary and the pantograph DOF. By applying the superposition principle, the solution of the pantograph dynamics at time-step $t$ can be split into:

$$
\mathbf{u}_{p}^{t}=\mathbf{u}_{i n i, p}^{t}+\mathbf{u}_{i n t, p}^{t}
$$

At this point, the contributions to the solution in Eqs. (24) and (28) coming from the non-linear force terms (i.e. the interaction and the dropper slackening correction forces) can be rewritten so that:

$$
\begin{aligned}
& \mathbf{q}_{c}^{t}=\mathbf{q}_{i n i, c}^{t}+f_{i n t}^{t} \tilde{\mathbf{q}}_{i n t, c}^{t}+\sum_{j}^{N_{s d}^{t}} f_{d j}^{t} \tilde{\mathbf{q}}_{\mathbf{d} j, c} \\
& \mathbf{u}_{p}^{t}=\mathbf{u}_{i n i, p}^{t}+f_{i n t}^{t} \tilde{\mathbf{u}}_{i n t, p}
\end{aligned}
$$

where $\tilde{\mathbf{q}}_{i n t, c}^{t}, \tilde{\mathbf{q}}_{\mathbf{d} j, c}$ and $\tilde{\mathbf{u}}_{i n t, p}$ are the displacements after one time-step $t$, produced by unitary forces acting on the contact point of the contact wire, on the slackened droppers and on the pantograph head, respectively. The values of the pantograph-catenary interaction force and the correction force of the $\mathrm{j}$-th slackened dropper, $f_{\text {int }}^{t}$ and $f_{d j}^{t}$, depend on $\mathbf{q}^{t}$ and have to be solved by setting a system of equations.

The seven terms present in the right hand side of Eq. (29) are the unknowns to be obtained to fulfil Eq. (21) along with Eqs. (2) and (6). The strategy used to compute them is based on performing as many calculations as possible in an offline stage, in which the computational cost is not a major issue, so that the subsequent online stage can be carried out in real-time.

\subsection{Offline calculations stage}

The offline stage of the method is devoted to performing all the calculations prior to the application of the time integration itself. In first place, the solution of the initial configuration problem, the solution of the eigenvalue problem for the catenary system and the modal basis projection are done in this initial stage.

However, the core of the offline stage is aimed at obtaining $\tilde{\mathbf{q}}_{i n t, c}^{t}, \tilde{\mathbf{q}}_{\mathbf{d} j, c}$ and $\tilde{\mathbf{u}}_{\text {int }, p}$ by 
solving single time-step problems with null initial conditions, in which a unitary load is applied on specific points of the models. These problems are:

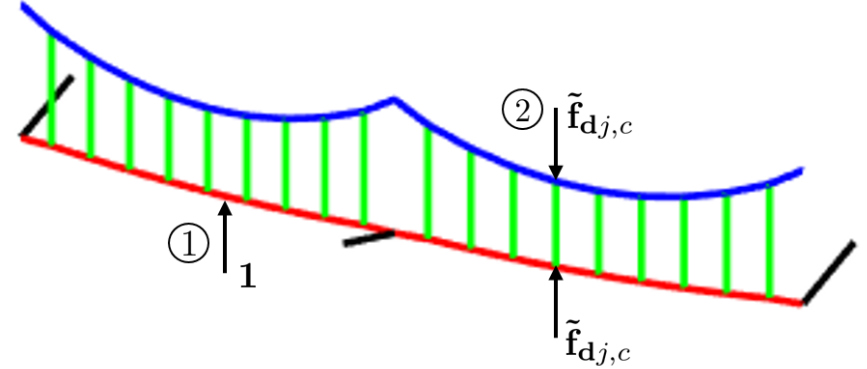

(a)

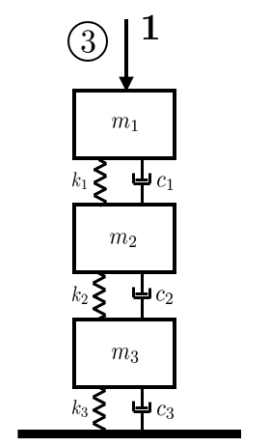

(b)

Figure 3: Unitary forces applied on the catenary (a), and the pantograph (b), to solve the single time-step problems in the offline stage.

1. Response of the catenary under a unitary load applied on the interaction point (see Figure 3a). Thus, as many problems as number of time-steps $N_{t}$ need to be solved. This set of problems has the following expression:

$$
\mathbf{K}_{I T c} \tilde{\mathbf{q}}_{i n t, c}^{t}=\Psi^{T} \tilde{\mathbf{F}}_{i n t, c}^{t}=\tilde{\mathbf{F}}_{i n t, c}^{t} \quad \text { for } \quad t=1, \ldots, N_{t}
$$

in which $\tilde{\mathbf{F}}_{\text {int,c }}^{t}$ results from expanding to the catenary problem size the unitary nodal force vector $\tilde{\mathbf{f}}_{i n t, c}^{t}=\mathbf{S}^{T}\left(\chi_{c w}\right) \cdot\left[\begin{array}{lll}0 & 0 & 1\end{array}\right]^{T}$. Generally, as depicted in Figure 4, the contact point does not match with a node, so that the shape functions matrix, $\mathbf{S}\left(\chi_{c w}\right)$, is used to relate the nodal displacements of the contact element with nodes $i$ and $j$ ( $\mathbf{u}_{i}^{t}$ and $\mathbf{u}_{j}^{t}$ respectively), with the displacements of the contact point with local coordinate $\chi_{c w}$ :

$$
\mathbf{u}_{c w}^{t}=\mathbf{S}\left(\chi_{c w}\right)\left[\begin{array}{c}
\mathbf{u}_{i}^{t} \\
\mathbf{u}_{j}^{t}
\end{array}\right]
$$

This matrix is composed of Hermitian polynomials which depend on the local coor- 
dinate $\chi$. That is:

$$
\begin{gathered}
\mathbf{S}(\chi)=\left[S_{1} \mathbf{I}_{3}\left|S_{2} \mathbf{I}_{3}\right| S_{3} \mathbf{I}_{3} \mid S_{4} \mathbf{I}_{3}\right] \\
S_{1}(\xi)=1-3 \xi^{2}+2 \xi^{3} \quad S_{2}(\xi)=l_{0}\left(\xi-2 \xi^{2}+\xi^{3}\right) \\
S_{3}(\xi)=3 \xi^{2}-2 \xi^{3} \quad S_{4}(\xi)=l_{0}\left(-\xi^{2}+\xi^{3}\right)
\end{gathered}
$$

in which the coordinate $\xi=\chi / l_{0} \in[0,1]$ denotes the normalised local coordinate for an element with undeformed length $l_{0}$, and $\mathbf{I}_{3}$ is the $3 \times 3$ identity matrix.

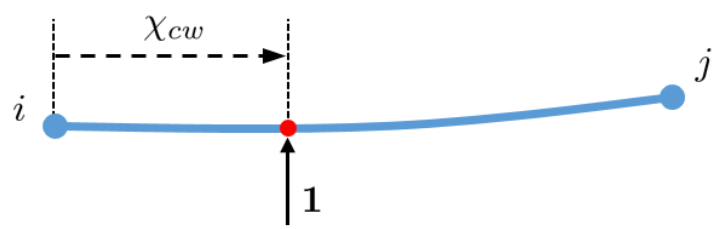

Figure 4: Unitary vertical force applied on the contact point of a contact cable element.

2. Response of the catenary under unitary compressive loads applied on the ends of each dropper and aligned with it (see Figure 3a). In this case, the problem reads:

$$
\mathbf{K}_{I T c} \tilde{\mathbf{q}}_{\mathbf{d} j, c}=\Psi^{T} \tilde{\mathbf{F}}_{\mathbf{d} j, c}=\tilde{\mathbf{F}}_{\mathbf{d} j, c} \quad \text { for } \quad j=1, \ldots, N_{d}
$$

which must be solved for any dropper of the catenary model. $\tilde{\mathbf{F}}_{\mathbf{d} j, c}$ is the result of assembling the nodal unitary force vectors $\tilde{\mathbf{f}}_{\mathbf{d} j, c}$ to the catenary model size.

3. Response of the pantograph under a unitary load applied on the top lumped mass (see Figure 3b). This problem is:

$$
\mathbf{K}_{I T p} \tilde{\mathbf{u}}_{i n t, p}=\tilde{\mathbf{F}}_{i n t, p}
$$

and is only solved once because the interaction force always acts on the same degree of freedom of the pantograph model. In this case, the force vector $\tilde{\mathbf{F}}_{\text {int,p }}=$ $\left[\begin{array}{llll}-1 & 0 & \ldots & 0\end{array}\right]^{T}$ includes as many elements as DOF of the pantograph model.

It is important to mention that not only the displacements of the previous problems are computed and stored, but also the modal velocities of the two problems related to the 
catenary are obtained following the Newmark rules:

$$
\begin{array}{lll}
\dot{\tilde{\mathbf{q}}}_{i n t, c}^{t}=b_{4} \tilde{\mathbf{q}}_{i n t, c}^{t} & \text { for } & t=1, \ldots, N_{t} \\
\dot{\tilde{\mathbf{q}}}_{\mathbf{d} j, c}=b_{4} \tilde{\mathbf{q}}_{\mathbf{d} j, c} & \text { for } & j=1, \ldots, N_{d}
\end{array}
$$

Regarding the computational cost of these problems, problem number 3 is solved with negligible computational cost since the pantograph model usually has a few DOF. However, the problems 1 and 2, which are related to the catenary, even though having a diagonal matrix $\mathbf{K}_{I T c}$, are more time-consuming because the force term has to be projected to the modal basis. Fortunately, this is not a big issue because this stage is performed offline, and the calculation speed is not particularly important.

Following with the idea of performing as many calculations as possible in the offline stage, there are other magnitudes that can be obtained before the time integration takes place, leading to a reduction in the number of operations performed in the online stage.

During the online stage (Section 2.4), the force of dropper $j$ will be evaluated as:

$$
f_{d j}^{t}=f_{d j, 0}+\left\langle\mathbf{k}_{\mathbf{d} j} \mathbf{u}_{\mathbf{d} j}^{t}+\mathbf{c}_{\mathbf{d} j} \dot{\mathbf{u}}_{\mathbf{d} j}^{t}\right\rangle=f_{d j, 0}+\left\langle\mathbf{k}_{\mathbf{d} j} \Psi_{\mathbf{d} j} \mathbf{q}_{c}^{t}+\mathbf{c}_{\mathbf{d} j} \Psi_{\mathbf{d} j} \dot{\mathbf{q}}_{c}^{t}\right\rangle
$$

where it is recalled that $\mathbf{u}_{\mathbf{d} j}^{t}$ is the nodal displacement vector of dropper $j$ and the operator $\langle\cdot\rangle$ extracts the force terms of the lower node and projects them to the direction of the dropper. Furthermore, $\boldsymbol{\Psi}_{\mathbf{d} j}$ is a submatrix composed of the six rows of $\boldsymbol{\Psi}$ linked to the DOF of the dropper element $j$.

If Eq. (29) is placed into (36), the force transmitted through dropper $j$, in time-step $t$, reads:

$$
\begin{aligned}
f_{d j}^{t}=f_{d j, 0}+\left\langle\mathbf{k}_{\mathbf{d} j} \Psi_{\mathbf{d} j}\left[\mathbf{q}_{i n i, c}^{t}+f_{i n t}^{t} \tilde{\mathbf{q}}_{i n t, c}^{t}+\sum_{r}^{N_{s d}^{t}} f_{d r}^{t} \tilde{\mathbf{q}}_{\mathbf{d} r, c}\right]\right\rangle+ \\
\left\langle\mathbf{c}_{\mathbf{d} j} \Psi_{\mathbf{d} j}\left[\dot{\mathbf{q}}_{i n i, c}^{t}+f_{i n t}^{t} \dot{\tilde{\mathbf{q}}}_{i n t, c}^{t}+\sum_{r}^{N_{s d}^{t}} f_{d r}^{t} \dot{\tilde{\mathbf{q}}}_{\mathbf{d} r, c}\right]\right\rangle
\end{aligned}
$$

where $\mathbf{q}_{i n i, c}^{t}, \tilde{\mathbf{q}}_{i n t, c}^{t}$ and $\tilde{\mathbf{q}}_{\mathbf{d} r, c}$ were defined in Eq. (29).

To speed up the calculation of expression (37) in the online stage, it is possible to pre- 
compute and store the following terms for each of the $N_{d}$ droppers of the model:

$$
\begin{array}{llll}
\mathbf{k}_{\mathbf{d} j}=\left\langle\mathbf{k}_{\mathbf{d} j} \boldsymbol{\Psi}_{\mathbf{d} j}\right\rangle & \mathbf{c}_{\mathbf{d} j}=\left\langle\mathbf{c}_{\mathbf{d} j} \boldsymbol{\Psi}_{\mathbf{d} j}\right\rangle & & \\
g_{i n t, j}^{t}=\mathbf{k}_{\mathbf{d} j} \tilde{\mathbf{q}}_{i n t, c}^{t} & \dot{g}_{i n t, j}^{t}=\mathbf{c}_{\mathbf{d} j} \dot{\tilde{\mathbf{q}}}_{i n t, c}^{t} & \text { for } & t=1, \ldots, N_{t} \\
g_{d j r}=\mathbf{k}_{\mathbf{d} j} \tilde{\mathbf{q}}_{\mathbf{d} r, c} & \dot{g}_{d j r}=\mathbf{c}_{\mathbf{d} j} \dot{\tilde{\mathbf{q}}}_{\mathbf{d} r, c} & \text { for } & r=1, \ldots, N_{d}
\end{array}
$$

The interaction force will also be evaluated in the online stage as defined in Eq. (4). In that expression, the vertical displacement of the contact point can be computed by the use of the third row of Eq. (31) and the modal transformation matrix, so that:

$$
u_{c w}^{t}=\left.\mathbf{S}\right|_{3}\left(\chi_{c w}\right)\left[\begin{array}{c}
\mathbf{u}_{i}^{t} \\
\mathbf{u}_{j}^{t}
\end{array}\right]=\left.\mathbf{S}\right|_{3}\left(\chi_{c w}\right) \Psi_{c w}^{t} \mathbf{q}_{c}^{t}
$$

in which $\Psi_{c w}^{t}$ is a submatrix composed of the 12 rows of $\boldsymbol{\Psi}$ related to the DOF of the contact cable element in which the interaction is taking place in time-step $t$, and $\left.\mathbf{S}\right|_{3}\left(\chi_{c w}\right)$ is the third row of the shape functions matrix (see Eq. (32)) evaluated in the local coordinate $\chi_{c w}$. With the vertical displacement of the contact point obtained from the modal coordinates, Eq. (29) can be placed into Eq. (4), leading to:

$$
f_{i n t}^{t}=f_{i n t 0}^{t}+k_{h}\left(u_{i n i, 1}^{t}+f_{i n t}^{t} \tilde{u}_{i n t, 1}-\left.\mathbf{S}\right|_{3}\left(\chi_{c w}\right) \Psi_{c w}^{t}\left[\mathbf{q}_{i n i, c}^{t}+f_{i n t}^{t} \tilde{\mathbf{q}}_{i n t, c}^{t}+\sum_{j}^{N_{s d}^{t}} f_{d j}^{t} \tilde{\mathbf{q}}_{\mathbf{d} j, c}\right]\right)
$$

From the previous expression, the magnitudes:

$$
\begin{aligned}
& \boldsymbol{\Upsilon}_{c w}^{t}=\left.\mathbf{S}\right|_{3}\left(\chi_{c w}\right) \mathbf{\Psi}_{c w}^{t} \\
& g_{i n t, c w}^{t}=k_{h} \boldsymbol{\Upsilon}_{c w}^{t} \tilde{\mathbf{q}}_{i n t, c}^{t} \\
& g_{i n t, d j}^{t}=k_{h} \boldsymbol{\Upsilon}_{c w}^{t} \tilde{\mathbf{q}}_{\mathbf{d} j, c} \quad \text { for } \quad j=1, \ldots, N_{d}
\end{aligned}
$$

can be calculated for each time-step of the simulation and stored to be used in the online stage of the method.

\subsection{Online time integration stage}

The time integration of Eq. (16) takes place in this stage of the simulation, in which a realtime solution is sought. Looking at the expressions (29), to obtain the nodal coordinates 
at time-step $t$, there are four terms which remain unknown, namely $\mathbf{q}_{i n i, c}^{t}, \mathbf{u}_{i n i, p}^{t}, f_{i n t}^{t}$, and $f_{d j}^{t}$ for $j=1, \ldots, N_{s d}^{t}$.

The two first terms can be available by solving the following systems of equations:

$$
\begin{aligned}
& \mathbf{K}_{I T c} \mathbf{q}_{i n i, c}^{t}=\mathbf{F}_{i n i, c}^{t}\left(\mathbf{q}_{c}^{t-1}, \dot{\mathbf{q}}_{c}^{t-1}, \ddot{\mathbf{q}}_{c}^{t-1}\right) \\
& \mathbf{K}_{I T p} \mathbf{u}_{i n i, p}^{t}=\mathbf{F}_{i n i, p}^{t}\left(\mathbf{u}_{p}^{t-1}, \dot{\mathbf{u}}_{p}^{t-1}, \ddot{\mathbf{u}}_{p}^{t-1}\right)
\end{aligned}
$$

The first equation related to the catenary is a set of decoupled equations since the matrix $\mathbf{K}_{I T c}$ is diagonal because of the use of modal coordinates for the catenary. This is a major advantage over the method proposed in [17] which allows the speeding up of the calculations. The second system of equations in (42), related to the pantograph, is solved quickly since it is composed only of a few number of equations.

Now, the values of the interaction force and the correction forces of each slackened dropper, $f_{i n t}^{t}$ and $f_{d j}^{t}$ for $j=1, \ldots, N_{s d}^{t}$, are the only variables that need to be obtained. The procedure followed to find them is based on setting and solving iteratively a small size system of linear equations. The first equation of that system comes from Eq. (40), which, after replacing the precomputed terms (41) and rearranging, becomes:

$$
\left(1-k_{h} \tilde{u}_{i n t, 1}+g_{i n t, c w}^{t}\right) f_{i n t}^{t}+\sum_{j}^{N_{s d}^{t}} g_{i n t, d j}^{t} f_{d j}^{t}=f_{i n t 0}^{t}+k_{h} \tilde{u}_{i n i, 1}^{t}-k_{h} \boldsymbol{\Upsilon}_{c w}^{t} \mathbf{q}_{i n i, c}^{t}
$$

The rest of the equations are deduced from Eq. (37), again after considering the precomputed magnitudes in the offline stage (see Eq. (38)) and performing some manipulations. The equation for the $j$-th slackened dropper, being $j=1, \ldots, N_{s d}^{t}$, reads as:

$$
\begin{array}{r}
-\left(g_{i n t, j}^{t}+\dot{g}_{i n t, j}^{t}\right) f_{i n t}^{t}-\left(g_{d j j}^{t}+\dot{g}_{d j j}^{t}+1\right) f_{d j}^{t}-\sum_{\substack{r \\
r \neq j}}^{N_{s d}^{t}}\left(g_{d j r}^{t}+\dot{g}_{d j r}^{t}\right) f_{d r}^{t}= \\
f_{d j, 0}+\mathbf{k}_{\mathbf{d} j} \mathbf{q}_{i n i, c}^{t}+\mathbf{c}_{\mathbf{d} j} \dot{\mathbf{q}}_{i n i, c}^{t}
\end{array}
$$

Eqs. (43) and (44) can be written in a matrix form to build the linear system of equations:

$$
\mathbf{A}^{t} \mathbf{f}_{n l}^{t}=\mathbf{b}^{t}
$$


where

$$
\begin{aligned}
& \mathbf{A}^{t}=\left(\begin{array}{cccc}
1-k_{h} \tilde{u}_{i n t, 1}+g_{i n t, c w}^{t} & g_{i n t, d 1}^{t} & g_{i n t, d 2}^{t} & \cdots \\
-g_{i n t, 1}^{t}-\dot{g}_{i n t, 1}^{t} & -g_{d 11}^{t}-\dot{g}_{d 11}^{t}-1 & -g_{d 12}^{t}-\dot{g}_{d 12}^{t} & \cdots \\
-g_{i n t, 2}^{t}-\dot{g}_{i n t, 2}^{t} & -g_{d 21}^{t}-\dot{g}_{d 21}^{t} & -g_{d 22}^{t}-\dot{g}_{d 22}^{t}-1 & \cdots \\
\vdots & \vdots & \ddots & \vdots \\
-g_{i n t, N_{s d}^{t}}^{t}+\dot{g}_{i n t, N_{s d}^{t}}^{t} & -g_{d N_{s d}^{t} 1}^{t}-\dot{g}_{d N_{s d}^{t} 1}^{t} & -g_{d N_{s d}^{t} 2}^{t}-\dot{g}_{d N_{s d}^{t} 2}^{t} & \cdots
\end{array}\right. \\
& \left.\begin{array}{cc}
\cdots & g_{i n t, d N_{s d}^{t}}^{t} \\
\cdots & -g_{d 1 N_{s d}^{t}-}^{t}-\dot{g}_{d 1 N_{s d}^{t}}^{t} \\
\cdots & -g_{d 2 N_{s d}^{t}}^{t}-\dot{g}_{d 2 N_{s d}^{t}}^{{ }^{t}} \\
\ddots & \vdots \\
\cdots & -g_{d N_{s d}^{t} N_{s d}^{t}-}^{t} \dot{g}_{d N_{s d}^{t} N_{s d}^{t}-1}^{t}
\end{array}\right) \\
& \mathbf{f}_{n l}^{t}=\left(\begin{array}{c}
f_{i n t}^{t} \\
f_{d 1}^{t} \\
f_{d 2}^{t} \\
\vdots \\
f_{d N_{s d}^{t}}^{t}
\end{array}\right) \quad \mathbf{b}^{t}=\left(\begin{array}{c}
f_{i n t 0}^{t}+k_{h}\left(\tilde{u}_{i n i, 1}^{t}-\boldsymbol{\Upsilon}_{c w}^{t} \mathbf{q}_{i n i, c}^{t}\right) \\
f_{d 1,0}+\mathbf{k}_{\mathbf{d} 1} \mathbf{q}_{i n i, c}^{t}+\mathbf{c}_{\mathbf{d} 1} \dot{\mathbf{q}}_{i n i, c}^{t} \\
f_{d 2,0}+\mathbf{k}_{\mathbf{d} 2} \mathbf{q}_{i n i, c}^{t}+\mathbf{c}_{\mathbf{d} 2} \dot{\mathbf{q}}_{i n i, c}^{t} \\
\vdots \\
f_{d N_{s d}^{t}, 0}+\mathbf{k}_{\mathbf{d} N_{s d}^{t}} \mathbf{q}_{i n i, c}^{t}+\mathbf{c}_{\mathbf{d} N_{s d}^{t} \dot{\mathbf{q}}_{i n i, c}^{t}}
\end{array}\right)
\end{aligned}
$$

The linear system (45) is set and solved at each time-step. After the first iteration, the non-linear contact loss criteria (Eq. (2)) is applied as well as the non-linear dropper slackening checking (Eq. (6)), to update the new slackened droppers $N_{s d}^{t}$ and therefore, reset the system (45). When two consecutive iterations provide the same results, the iterative process is finished and the time integration procedure can be moved to the next time-step.

The most demanding tasks in each time-step of the proposed algorithm, from the computation cost point of view, are evaluating the force transmitted by all the droppers of the model according to Eq. (2), since it implies performing the product $\mathbf{k}_{\mathbf{d} j} \mathbf{q}_{c}^{t}$ for $j=1, \ldots, N_{d}$, and particularising on the contact point the response of the catenary to the initial conditions, by the product $\boldsymbol{\Upsilon}_{c w}^{t} \mathbf{q}_{i n i, c}^{t}$.

Despite this issue, it is important to highlight that the system (45) is very reduced in size 
(usually about 2 to 5 equations) and besides, it rarely needs more than three iterations to be solved. Definitely, these features make this approach a very efficient way to deal with the non-linearities present in the catenary-pantograph dynamic interaction simulation. Apart from efficiency, another of the advantages of the method is that no additional assumptions are made with respect to the direct method explained in Section 2.1. Thus, the accuracy of the results is not affected.

For the hypothetical use of this algorithm to perform hybrid simulations, in which the pantograph model is replaced by the real device, some modifications in the formulation are proposed to adapt the algorithm to this scenario (see Section 3). Another important issue is regarding the modal basis size. If it is reduced by modal truncation, the computational cost of the products $\mathbf{k}_{\mathbf{d} j} \mathbf{q}_{c}^{t}$ and $\boldsymbol{\Upsilon}_{c w}^{t} \mathbf{q}_{i n i, c}^{t}$ could be alleviated. A good balance between speed up of calculations and accuracy of the results is sought in Section 4.

\section{Explicit treatment of the interaction force}

To obtain the dynamic response of the catenary, Eq. (21) must be solved, in which right hand side of Eq. (22) is composed of three force contributions, namely:

$$
\mathbf{F}_{I T c}^{t}=\mathbf{F}_{i n i, c}^{t}\left(\mathbf{q}_{c}^{t-1}\right)+\mathbf{F}_{i n t, c}^{t}\left(\mathbf{q}^{t}\right)+\mathbf{F}_{\mathbf{d}, c}^{t}\left(\mathbf{q}_{c}^{t}\right)
$$

In this work, replacing the interaction force, $\mathbf{F}_{\text {int,c }}^{t}\left(\mathbf{q}^{t}\right.$ ) (which couples both the catenary and the pantograph DOF), by its value in the previous time-step $\mathbf{F}_{i n t, c}^{t}\left(\mathbf{q}^{t-1}\right)$, it is proposed. This approach entails incorporating the interaction force explicitly into the time integration scheme. Thus, the right hand side of Eq. (22) becomes:

$$
\mathbf{F}_{I T c}^{t}=\mathbf{F}_{i n i, c}^{t}\left(\mathbf{q}_{c}^{t-1}\right)+\mathbf{F}_{i n t, c}^{t-1}+\mathbf{F}_{\mathbf{d}, c}^{t}\left(\mathbf{q}_{c}^{t}\right)
$$

and the unknown modal coordinates can be split into:

$$
\mathbf{q}_{c}^{t}=\mathbf{q}_{i n i, c}^{t}+f_{i n t}^{t-1} \tilde{\mathbf{q}}_{i n t, c}^{t}+\sum_{j}^{N_{s d}^{t}} f_{d j}^{t} \tilde{\mathbf{q}}_{\mathbf{d} j, c}
$$


where the interaction force $f_{i n t}^{t-1}$ is known at the current time-step and $\tilde{\mathbf{q}}_{i n t, c}^{t}$ and $\tilde{\mathbf{q}}_{\mathbf{d} j, c}$ are obtained by solving problems 1 and 2 of the offline stage.

Regarding the calculations in the online stage, $\mathbf{q}_{i n i, c}^{t}$ is computed with the first expression in Eq. (42). The rest of the unknowns, the slackened droppers correction forces $f_{d j}^{t}$ for $j=1, \ldots, N_{s d}^{t}$, are now obtained by setting and solving iteratively a new version of the system (45), in which now:

$$
\begin{aligned}
& \mathbf{A}^{t}=\left(\begin{array}{cccc}
-g_{d 11}^{t}-\dot{g}_{d 11}^{t}-1 & -g_{d 12}^{t}-\dot{g}_{d 12}^{t} & \cdots & -g_{d 1 N_{s d}^{t}}^{t}-\dot{g}_{d, 1 N_{s d}^{t}}^{t} \\
-g_{d 21}^{t}-\dot{g}_{d 21}^{t} & -g_{d 22}^{t}-\dot{g}_{d 22}^{t}-1 & \cdots & -g_{d 2 N_{s d}^{t}}^{t}-\dot{g}_{d 2 N_{s d}^{t}}^{t} \\
\vdots & \vdots & \ddots & \vdots \\
-g_{d N_{s d}^{t} 1}^{t}-\dot{g}_{d N_{s d}^{t} 1}^{t} & -g_{d N_{s d}^{t} 2}^{t}-\dot{g}_{d N_{s d}^{t} 2}^{t} & \cdots & -g_{d N_{s d}^{t} N_{s d}^{t}}^{t}-\dot{g}_{d N_{s d}^{t} N_{s d}^{t}}^{t}-1
\end{array}\right) \\
& \mathbf{f}_{n l}^{t}=\left(\begin{array}{c}
f_{d 1}^{t} \\
f_{d 2}^{t} \\
\vdots \\
f_{d N_{s d}^{t}}^{t}
\end{array}\right) \mathbf{b}^{t}=\left(\begin{array}{c}
f_{d 1,0}+\mathbf{k}_{\mathbf{d} 1} \mathbf{q}_{i n i, c}^{t}+\mathbf{c}_{\mathbf{d} 1} \dot{\mathbf{q}}_{i n i, c}^{t}+f_{i n t}^{t-1}\left(g_{i n t, 1}^{t}+\dot{g}_{i n t, 1}^{t}\right) \\
f_{d 2,0}+\mathbf{k}_{\mathbf{d} 2} \mathbf{q}_{i n i, c}^{t}+\mathbf{c}_{\mathbf{d} 2} \dot{\mathbf{q}}_{i n i, c}^{t}+f_{i n t}^{t-1}\left(g_{i n t, 2}^{t}+\dot{g}_{i n t, 2}^{t}\right) \\
\vdots \\
f_{d N_{s d}^{t}, 0}+\mathbf{k}_{\mathbf{d} N_{s d}^{t}} \mathbf{q}_{i n i, c}^{t}+\mathbf{c}_{\mathbf{d} N_{s d}^{t}} \dot{\mathbf{q}}_{i n i, c}^{t}+f_{i n t}^{t-1}\left(g_{i n t, N_{s d}^{t}}^{t}+\dot{g}_{i n t, N_{s d}^{t}}^{t}\right)
\end{array}\right)
\end{aligned}
$$

Remark. From a practical point of view the matrix $\mathbf{A}^{t}$ can be considered a diagonal matrix for nonstitched catenaries because the coefficients $g_{d j r}^{t}$ and $\dot{g}_{d j r}^{t}$ are negligible if $j \neq r$. This assumption does not degrade the accuracy of the simulations.

After each solution of this system of equations, the slackening criterion (Eq. (6)) is applied and the system is reset again with the new slackened droppers until two consecutive iterations provide the same results. At this point, $\mathbf{q}_{c}^{t}$ is computed, and it is necessary to obtain the vertical displacement of the contact wire on the interaction point as:

$$
u_{c w}^{t}=\boldsymbol{\Upsilon}_{c w}^{t} \mathbf{q}_{c}^{t}
$$

where $\boldsymbol{\Upsilon}_{c w}^{t}$ has been defined in Eq. (41). With this value, the dynamics of the pantograph model can be solved and the new $f_{\text {int }}^{t}$ obtained, which will be feed into the catenary model in the next time-step of the algorithm.

To investigate the effect on the results produced by the explicit treatment of the interaction 
force, a numerical experiment is carried out. The catenary and the pantograph models used in all the examples of this work are those employed in the benchmark exercise [4]. Although some authors extend their analyses up to $100 \mathrm{~Hz}$ [20, 22, 23], this requires to consider enhanced pantograph models which allow for flexibility modes and, it is out of the scope of the present work. Thus, the dynamic behaviour of the system is only analysed up to $20 \mathrm{~Hz}$, following the guidelines of current standards. The simulation is performed for $10 \mathrm{~s}$, with a time-step $\Delta t=0.001 \mathrm{~s}$, a penalty stiffness $k_{h}=50000 \mathrm{~N} / \mathrm{m}$ [3], and the pantograph is running at $300 \mathrm{~km} / \mathrm{h}$. The $20 \mathrm{~Hz}$ filtered interaction force obtained is shown in Figure 5 with a zoom window comprising the interval from 4 to 6 s.

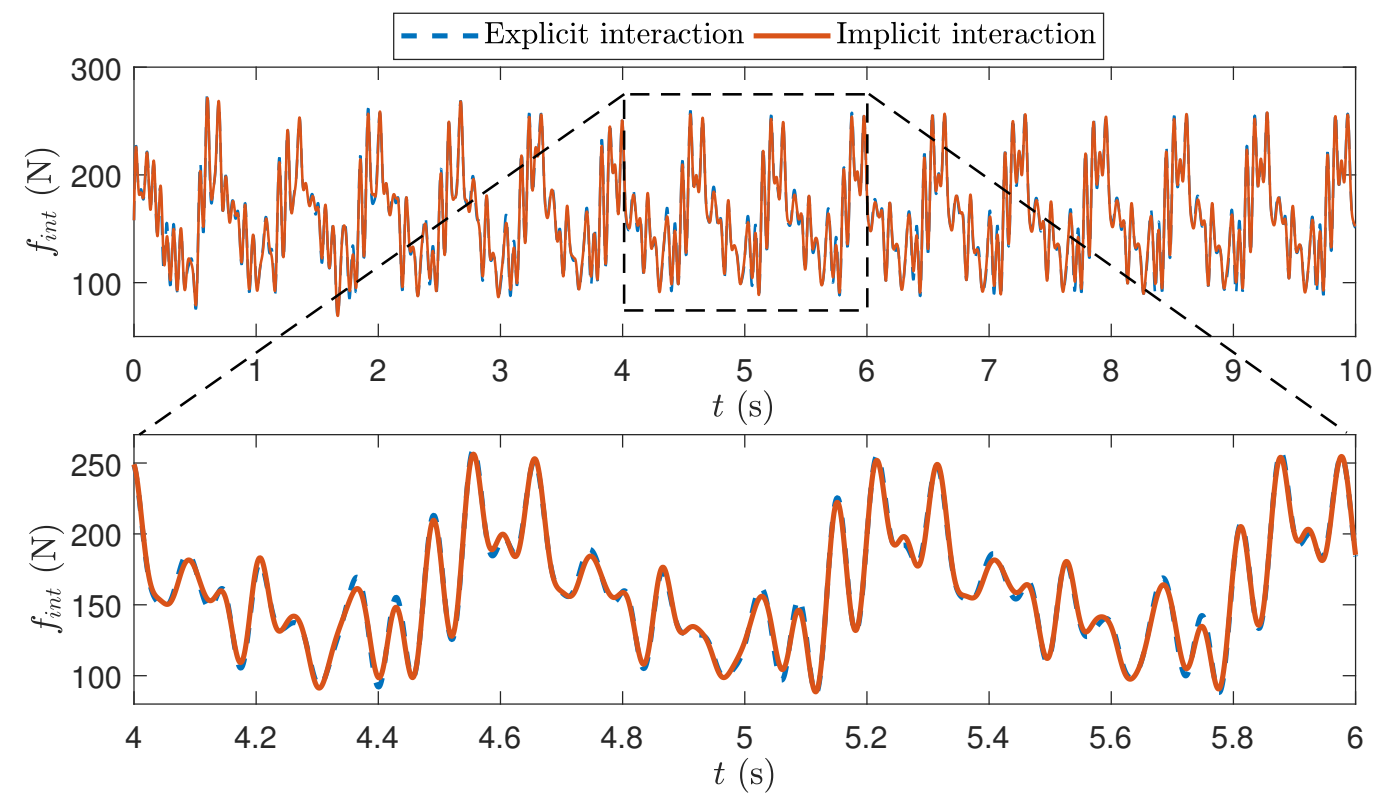

Figure 5: Comparison of the $20 \mathrm{~Hz}$ filtered interaction force when treated explicitly (dashed line) and implicitly (solid line) in the time integration process. Zoom window from 4 to $6 \mathrm{~s}$ (bottom figure).

\begin{tabular}{cccc} 
& Implicit interaction & Explicit interaction & Relative error \\
\hline $\bar{f}_{\text {int }}$ & $159.22 \mathrm{~N}$ & $159.20 \mathrm{~N}$ & $0.013 \%$ \\
\hline$\sigma\left(f_{\text {int }}\right)$ & $40.33 \mathrm{~N}$ & $41.23 \mathrm{~N}$ & $2.23 \%$ \\
\hline $\max \left(f_{\text {int }}\right)$ & $256.76 \mathrm{~N}$ & $259.62 \mathrm{~N}$ & $1.11 \%$ \\
\hline $\min \left(f_{\text {int }}\right)$ & $88.58 \mathrm{~N}$ & $86.39 \mathrm{~N}$ & $2.47 \%$ \\
\hline
\end{tabular}

Table 1: Comparison of statistical values of the interaction force interval from 4 to 8 seconds, resulting from implicit and explicit treatment. 
At first glance good agreement is observed between the results obtained with an implicit (solid line) and an explicit (dashed line) treatment of the interaction force. Before stabilising, during the first four seconds the interaction force shows a transient regime related to start-up effects. To quantify the difference between any two discrete signals $x_{1}(t)$ and $x_{2}(t)$ with $N_{t}$ points each, the coefficient of variation $(\mathrm{CV})$ of the root mean square error (RMSE) is defined as:

$$
C V=\frac{R M S E}{\bar{x}_{1}} \times 100=\frac{\sqrt{\frac{\sum_{t=1}^{N_{t}}\left(x_{1}^{t}-x_{2}^{t}\right)^{2}}{N_{t}}}}{\bar{x}_{1}} \times 100
$$

where $\bar{x}_{1}$ is the mean value of $x_{1}(t)$.

Applying this measure to the explicit and the implicit integrated interaction forces shown in Figure 5, a CV of $1.97 \%$ is obtained. This low CV is reaffirmed by the similarity in the statistical values presented in Table 1 . This results confirm the validity of the explicit treatment of the interaction force to perform pantograph-catenary dynamic interaction simulations and opens the possibility of using this algorithm in HIL simulations, in which the value of the contact force at the previous time-step $f_{\text {int }}^{t-1}$, needed to feed the catenary model, would be measured in the test rig.

\section{Modal basis truncation}

Usually, when projecting a set of coordinates to a modal basis, it is possible to truncate this basis to reduce the dimension of the modal space without affecting substantially the accuracy of the results. Therefore, the $r$-th nodal DOF of the catenary can be approximated by:

$$
u_{c, r}^{t} \approx \bar{u}_{c, r}^{t}=\sum_{i=1}^{M} \Psi_{r, i} q_{c, i}^{t} \quad \rightarrow \quad \mathbf{u}_{c}^{t} \approx \overline{\mathbf{u}}_{c}^{t}=\overline{\mathbf{\Psi}} \mathbf{q}_{c}^{t}
$$

where $\overline{\boldsymbol{\Psi}}$ is the truncated modal transformation matrix of size $N \times M, N$ being the number of DOF of the catenary model and $M$ the number of selected modes. If $M<<N$, the proposed algorithm is even more efficient from the computational point of view, because the computations with vectors and matrices whose size is equal to the number of modal coordinates, entail less computation effort. 
To select a priori the $M$ most relevant modes, it is usual to keep the modes associated with the lowest natural frequencies and discard those related to higher frequency content. Following this criterion, the $20 \mathrm{~Hz}$ filtered interaction force obtained with different sizes of the modal basis are compared in Figure 6. Although the catenary model used (see [4]) has $N=23966 \mathrm{DOF}$, the use of $M=15000$ modes guarantees the accuracy of the interaction force. This implies keeping modes with natural frequencies up to $342 \mathrm{~Hz}$, as shown in Table 2.

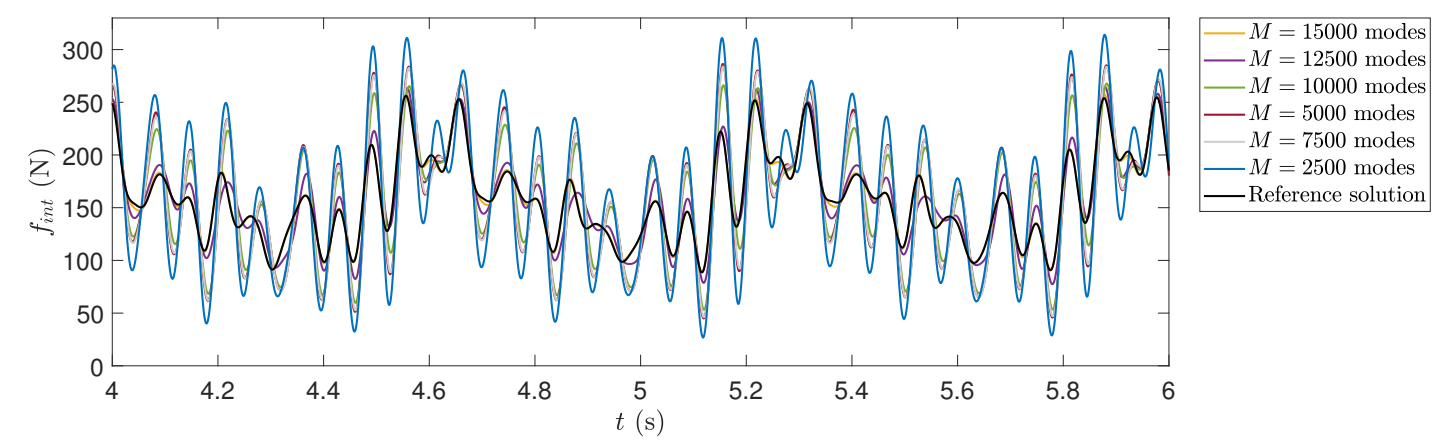

Figure 6: $20 \mathrm{~Hz}$ filtered interaction force for different levels of modal basis truncation, following the frequency criterion.

With the aim of reducing the required modes, a different truncation criterion is proposed. Assuming that the interaction force mainly depends on the vertical movement of the contact cable, a selection of the modes with the most contribution to the vertical displacement of the cables is sought. To this end, the parameter $\kappa_{z}$ is defined for the mode $i$ as:

$$
\kappa_{z}^{i}=\frac{1}{\omega_{i}} \boldsymbol{\Psi}_{z, i}^{T} \cdot \Psi_{z, i}
$$

where $\omega_{i}$ is the natural frequency of the mode $i$, and $\boldsymbol{\Psi}_{z, i}$ is a subset of the eigenmode $\boldsymbol{\Psi}_{i}$, composed of the elements related to the DOF that contribute to the vertical movement of the catenary cables. After ordering the modes from the highest to the lowest $\kappa_{z}$, the $20 \mathrm{~Hz}$ filtered interaction force obtained when keeping different numbers of modes $M$, are compared in Figure 7. In this case, a good accuracy remains until the number of selected modes decreases from $M=10000$. However, the maximum frequency content of the truncated modal basis is about $360 \mathrm{~Hz}$ (see Table 2), which is very similar to that found when the frequency truncation criterion was used.

To quantify the truncation error, the values of the CV of the RMSE between the refer- 


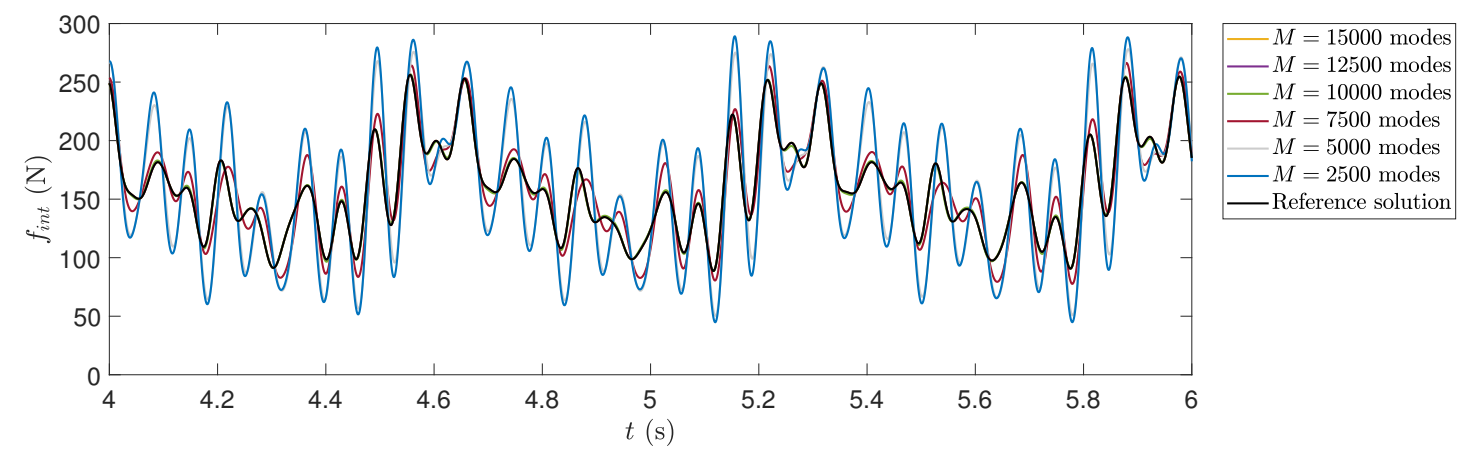

Figure 7: $20 \mathrm{~Hz}$ filtered interaction force for different levels of modal basis truncation, following the vertical displacement criterion.

\begin{tabular}{|c|c|c|c|c|}
\hline \multirow[b]{3}{*}{$M$} & \multicolumn{4}{|c|}{ Truncation criterion } \\
\hline & \multicolumn{2}{|c|}{ Frequency } & \multicolumn{2}{|c|}{ Vertical displacement } \\
\hline & $f_{\max }(\mathrm{Hz})$ & $\mathrm{CV}(\mathrm{RMSE})(\%)$ & $f_{\max }(\mathrm{Hz})$ & $\mathrm{CV}(\mathrm{RMSE})$ \\
\hline 15000 & 342.8 & 1.00 & 723.9 & 0.004 \\
\hline 12500 & 249.3 & 7.84 & 373.1 & 0.03 \\
\hline 10000 & 172.6 & 18.22 & 358.1 & 0.64 \\
\hline 7500 & 118.1 & 21.37 & 257.2 & 8.60 \\
\hline 5000 & 73.9 & 21.37 & 152.3 & 20.08 \\
\hline 2500 & 36.1 & 28.07 & 66.12 & 21.85 \\
\hline
\end{tabular}

Table 2: Maximum natural frequency and coefficient of variation of the RMSE for both the frequency and the vertical displacement-based truncation criteria.

ence and the truncated solutions, listed on Table 2, are plotted in Figure 8 for the two truncation criteria considered. It is clearly noticed that the vertical displacement modal truncation is more effective than the truncation based on the frequency content since the modes associated with the lateral and longitudinal displacements do not contribute significantly to the dynamic response of the catenary.

Even so, in the best scenario to provide accurate results (vertical displacement truncation with $M=10000$ ), the number of required modes seems to be excessive if comparing the frequency content of the analysed results (up to $20 \mathrm{~Hz}$ ) with the natural frequency of the influential modes (up to $360 \mathrm{~Hz}$ ). This issue has been noticed only if the non-linearity of dropper slackening is considered and it is caused by the inability of the chosen modal basis to represent the low frequency response of the catenary without including modes 


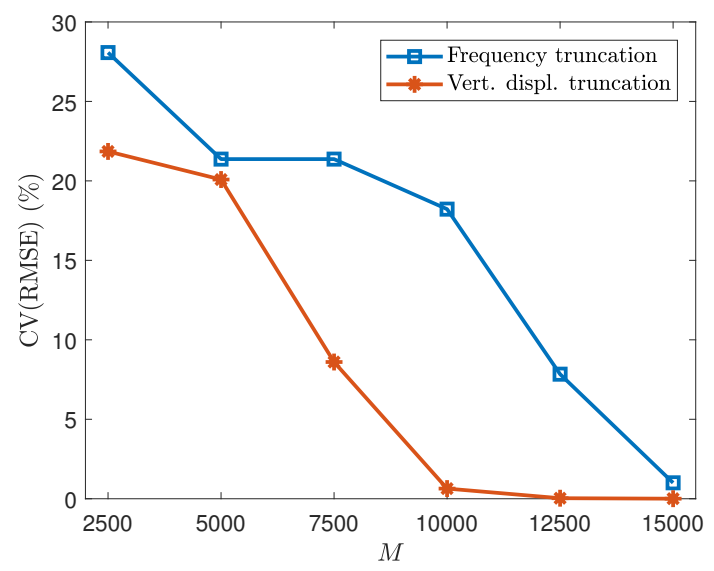

Figure 8: $C V(R M S E)$ as a function of the truncated modal basis size for both the frequency and the vertical displacement truncation criteria.

with high frequency content.

To point out this feature, the results shown in Figure 7 are recomputed with a catenary model whose droppers behave linearly, i.e. they do not slacken under compression. If the modal basis is truncated according to the $\kappa_{z}$ value of each mode, the $20 \mathrm{~Hz}$ filtered interaction forces obtained with different truncation levels are plotted in Figure 9. The good accuracy of the results can be appreciated if dropper slackening is not considered, even with a modal basis composed of only $M=2000$ modes and a maximum frequency content around $50.5 \mathrm{~Hz}$.

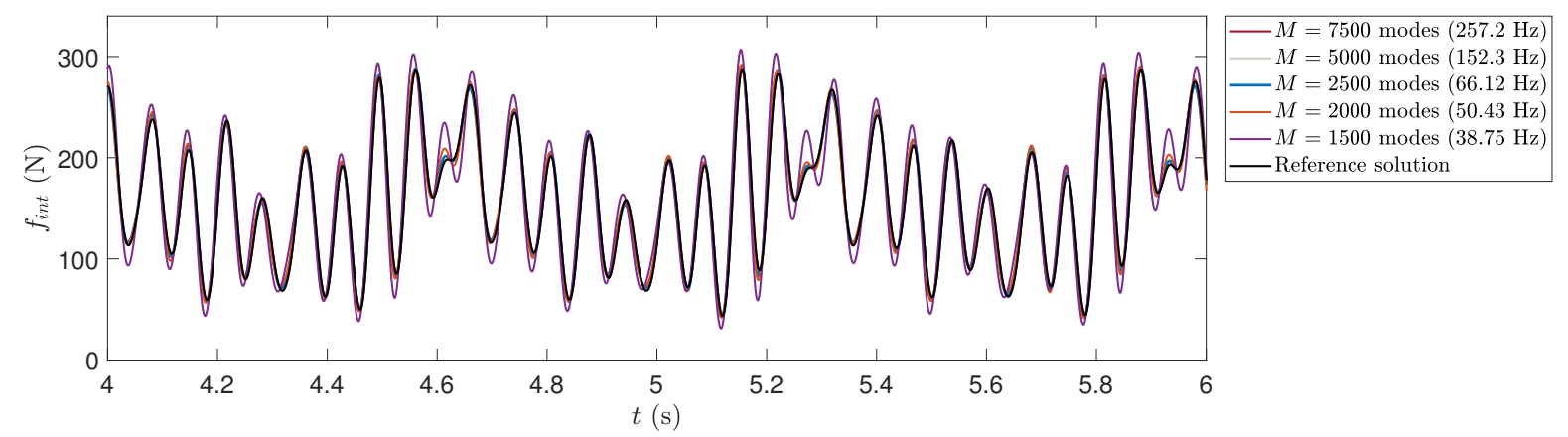

Figure 9: $20 \mathrm{~Hz}$ filtered interaction force for different levels of modal basis truncation, when linear dropper behaviour is assumed.

To strengthen this conclusion, the CV of the RMSE between the reference and the truncated solutions is compared in Figure 10. It is remarkable to see the great reduction in the number of modes necessary to obtain an accurate solution when the problem turned 
linear. Specifically, $M=5000$ modes $(150 \mathrm{~Hz})$ are enough to obtain an interaction force with similar accuracy to that obtained with $M=10000$ modes $(358.1 \mathrm{~Hz})$ when dropper slackening is considered.

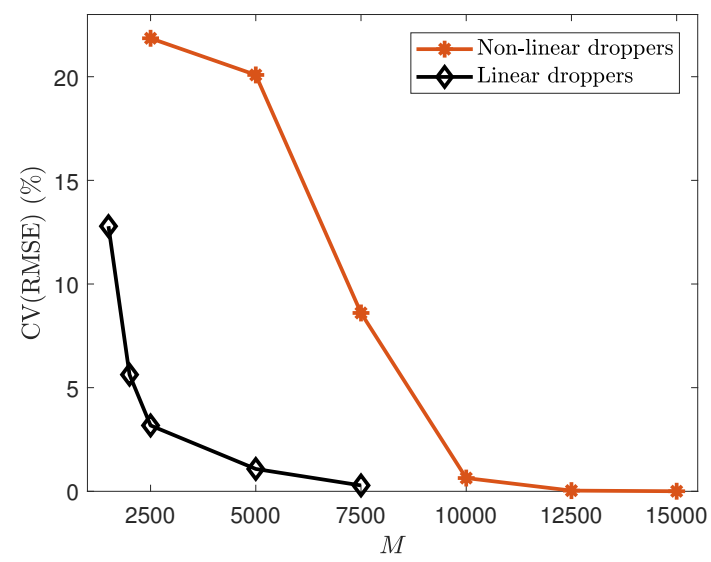

Figure 10: Evolution of the $C V(R M S E)$ with the truncated modal basis size when non-linear and linear droppers are considered in the catenary model.

A way to check if the poor performance of the modal basis used when a dropper is slackened, consists of projecting the modes of a catenary with one dropper missing to the reference modal basis. To this end, a single span catenary model is used without loss of generality. When the pantograph pushes up, for example, the fourth dropper, it slackens and the catenary behaves as if this dropper were missing. Thus, the modes of the catenary model without the fourth dropper, $\Pi_{n}$ for $n=1, \ldots, N^{\prime}$, can be calculated and projected to the modal basis $\boldsymbol{\Psi}$ by solving the following system of equations:

$$
\Psi \boldsymbol{\eta}_{n}=\Pi_{n}
$$

where $\boldsymbol{\eta}_{n}$ is the vector of weights to express the vector $\boldsymbol{\Pi}_{n}$ as a linear combination of the vectors $\boldsymbol{\Psi}_{n}$.

In Figure 11a, the mode $\Pi_{14}$ with a natural frequency of $5.3 \mathrm{~Hz}$, is depicted while the mode $\Pi_{53}$, with a natural frequency of $16.1 \mathrm{~Hz}$, is plotted in Figure 11b. The absolute value of the weights $\boldsymbol{\eta}_{n}$ obtained after solving Eq. (56) for $n=14$ and $n=53$, are shown in Figure 12. This last graph shows the need to consider high frequency modes (up to $300 \mathrm{~Hz}$ ) of the full catenary model to represent low frequency modes $(5.3$ and $16.1 \mathrm{~Hz})$ of the same catenary with the lack of one dropper, i.e. with a slackened dropper. 


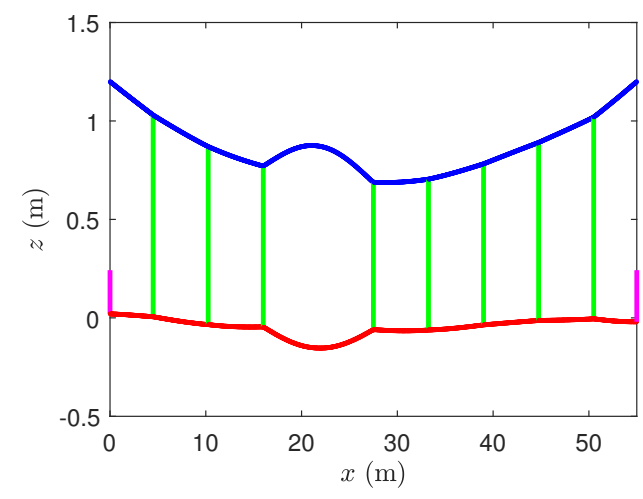

(a)

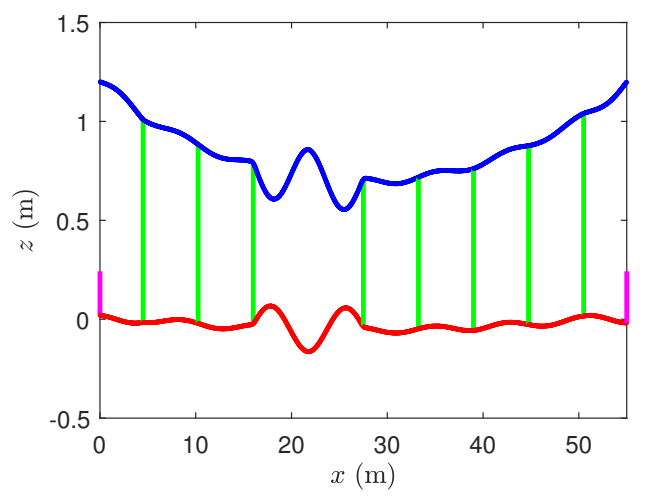

(b)

Figure 11: Graphical representation of the $14^{\text {th }}$ (a), and the $53^{\text {rd }}$ (b) modes for a catenary with the fourth dropper missing.

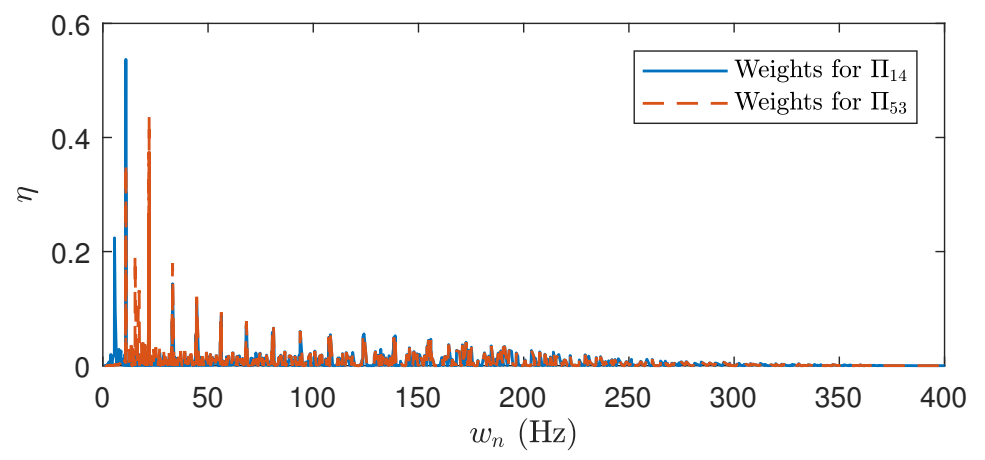

Figure 12: Absolute value of the projection weights $\boldsymbol{\eta}_{14}$ (solid line) and $\boldsymbol{\eta}_{53}$ (dashed line).

\section{Study of the real-time capabilities}

In this section, the computational time required to perform numerical simulations is analysed. The algorithm is coded in MATLAB ${ }^{\circledR}$ and carried out in an Intel ${ }^{\circledR} \mathrm{Xeon}^{\circledR}$ ES-1660 v3 CPU.

The first analysis is focused on unveiling the relationship between the number of modes considered in the modal basis $M$, and the computational cost of the simulations $t_{\text {comp }}$ considering the online stage, in which the time integration takes place. To this end, simulations for $11 \mathrm{~s}$ of a twenty-span catenary model interacting with a pantograph travelling at $300 \mathrm{~km} / \mathrm{h}$ are performed. 


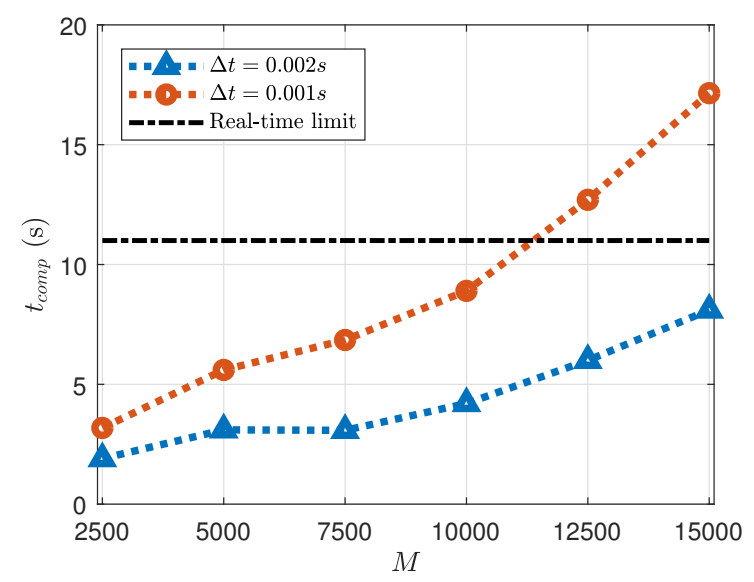

Figure 13: Computational time versus the number of modes $M$ of the truncated modal basis when the time integration is carried out with $1 \mathrm{~ms}$ (circles) and $2 \mathrm{~ms}$ (triangles) time-step.

The evolution of the computational cost with the modal basis size is shown in Figure 13. The study is made for two different time steps, namely $\Delta t=0.001 \mathrm{~s}$ and $\Delta t=0.002 \mathrm{~s}$. The computational effort necessary in both scenarios increases almost linearly with the number of modes considered, $M$. By using $\Delta t=0.001 \mathrm{~s}$, simulations with more than 11000 modes exceed the real-time limit (11 s). However, as demonstrated in Section 4, $M=10000$ modes suffice to obtain accurate results while keeping real-time performance. A higher time-step, $\Delta t=0.002 \mathrm{~s}$, results in good accuracy (see [17]) and the real-time limit is not overcome for any number of modes. These features enable the use of the proposed algorithm in an HIL set-up, in which the catenary is replaced by a virtual model.

The second study is aimed at comparing the efficiency of the proposed method, which considers a modal approach, with the offline/online strategy published in [17]. Four catenary models, with 5, 10, 15 and 20 spans have been used to perform simulations of 2 , 5,8 and 11 s respectively.

The computational time required to perform such simulations is shown in Table 3. It is important to remark that the size of the modal basis $M$ used for each catenary model has been tuned for the CV(RMSE) of the $20 \mathrm{~Hz}$ filtered interaction force to be around $1 \%$. The main conclusion drawn from this study of the computational cost is the notable speed-up factor $(\sim 5)$ achieved with the modal approach combined with the offline/online integration strategy. Thus, the presented method provides the necessary features to perform HIL simulations in contrast to those provided by the initial offline/online strategy 


\begin{tabular}{rcccc} 
& 5 spans & 10 spans & 15 spans & 20 spans \\
\hline Simulated time (s) & 2 & 5 & 8 & 11 \\
\hline Online stage in [17] (s) & $\mathbf{1 . 0 8}$ & $\mathbf{5 . 4 3}$ & $\mathbf{1 3 . 7 0}$ & $\mathbf{2 4 . 5 9}$ \\
\hline Proposed algorithm (s) & $\mathbf{0 . 2 1}$ & $\mathbf{1 . 0 6}$ & $\mathbf{2 . 4 5}$ & $\mathbf{5 . 6 7}$ \\
\hline $\mathrm{M}$ & 2500 & 5000 & 7500 & 10000 \\
\hline CV (RMSE) (\%) & 1.26 & 1.10 & 0.92 & 0.90 \\
\hline Speed-up & $\mathbf{5 . 1 4}$ & $\mathbf{5 . 1 2}$ & $\mathbf{5 . 5 9}$ & $\mathbf{4 . 3 4}$ \\
\hline
\end{tabular}

Table 3: Computational cost comparison between the proposed real-time algorithm and the online stage of the method presented in [17].

presented in [17].

\section{Conclusions}

In this work, a strategy to simulate the pantograph-catenary dynamic interaction in realtime has been proposed. The method allows performing HIL simulations in which the catenary is replaced by a numerical model. Based on the efficient approach presented in [17], the projection of the catenary coordinates to a modal basis is proposed. This leads to a diagonal matrix in each step of the time integration method, which allows to speed up the calculations notably.

The integration strategy has been adapted to deal with the interaction force in an explicit way, by decoupling the pantograph and catenary models. This could be used in a real HIL test in which the contact comes from the measure provided by the sensors located between the linear actuator and the pantograph contact strips. The results corroborate that this decoupling can be made without introducing significant differences in the simulation results with respect to those obtained for the coupled system, which is fully treated implicitly.

With the aim of keeping the computational cost as low as possible, the truncation of the modal basis has been studied. It has been shown that the best criterion to carry out the modal basis truncation is that based on the vertical displacement contribution of each 
mode. However, the number of required modes to keep a good accuracy of the results seemed too high for the frequency range of interest. According to our simulations, it has been concluded that this phenomenon occurs due to the poor ability of the modal basis to represent configurations with slackened droppers.

Regarding the computational cost, simulations with both the coupled implicit and the uncoupled explicit strategies require a very similar computational time. However, when compared with the offline/online strategy proposed in [17], a speed-up factor around 5 is accomplished with the use of modal coordinates.

\section{Acknowledgements}

The authors would like to acknowledge the funding provided by the Regional Government of Valencia (PROMETEO/2016/007) and the Spanish Ministry of Economy, Industry and Competitiveness (TRA2017-84736-R).

\section{Conflict of interest}

The authors declare that they have no conflict of interest.

\section{References}

[1] G. Poetsch, J. Evans, R. Meisinger, W. Kortüm, W. Baldauf, A. Veitl, and J. Wallaschek, "Pantograph/catenary dynamics and control," Vehicle System Dynamics, vol. 28, no. 2-3, pp. 159-195, 1997.

[2] F. Kiessling, R. Puschmann, and A. Schmieder, Contact lines for electrical railways: planning-design-implementation. Erlangen: Publicis Corporate Publishing, 2002. 
[3] UNE 50318, "Railway applications. Current collection systems. Validation of simulation of the dynamic interaction between pantograph and overhead contact line," European Committee for Electrotechnical Standarization, 2002.

[4] S. Bruni, J. Ambrósio, A. Carnicero, Y. H. Cho, L. Finner, M. Ikeda, S. Y. Kwon, J. P. Massat, S. Stichel, and M. Tur, "The results of the pantograph-catenary interaction benchmark," Vehicle System Dynamics, vol. 53, no. 3, pp. 412-435, 2015.

[5] P. H. Poznic, J. Jerrelind, and L. Drugge, "Experimental evaluation of nonlinear dynamics and coupled motions in a pantograph," in ASME 2009 International Design Engineering Technical Conferences and Computers and Information in Engineering Conference, pp. 619-626, American Society of Mechanical Engineers, 2009.

[6] J. Ambrósio, F. Rauter, J. Pombo, and M. Pereira, "A flexible multibody pantograph model for the analysis of the catenary-pantograph contact," Multibody Dynamics, vol. 23, pp. 1-27, 2011.

[7] A. Collina and A. L. C. M. Carnevale, "Effect of collector deformable modes in pantograph-catenary dynamic interaction," Proceedings of the Institution of Mechanical Engineers, Part F: Journal of Rail and Rapid Transit, vol. 223, no. 1, pp. 1-14, 2009.

[8] "PantoTRAIN report summary. Pantograph and catenary interaction total regulatory acceptance for the interoperable network," Union des Industries Ferroviaires Européennes - UNIFE, Brussels, Belgium, 2013.

[9] W. Zhang, G. Mei, X. Wu, and Z. Shen, "Hybrid simulation of dynamics for the pantograph-catenary system," Vehicle System Dynamics, vol. 38, no. 6, pp. 393-414, 2002.

[10] F. Resta, A. Facchinetti, A. Collina, and G. Bucca, "On the use of a hardware in the loop set-up for pantograph dynamics evaluation," Vehicle System Dynamics, vol. 46, no. S1, pp. 1039-1052, 2008.

[11] A. Facchinetti and M. Mauri, "Hardware-in-the-loop overhead line emulator for active pantograph testing," IEEE Transactions on Industrial Electronics, vol. 56, no. 10, pp. 4071-4078, 2009. 
[12] S. Bruni, G. Bucca, A. Collina, and A. Facchinetti, "Numerical and hardware-in-theloop tools for the design of very high speed pantograph-catenary systems," Journal of Computational and Nonlinear Dynamics, vol. 7, no. 4, p. 041013, 2012.

[13] A. Facchinetti and S. Bruni, "Hardware-in-the-loop hybrid simulation of pantographcatenary interaction," Journal of Sound and Vibration, vol. 331, no. 12, pp. 27832797, 2012 .

[14] A. Facchinetti, L. Gasparetto, and S. Bruni, "Real-time catenary models for the hardware-in-the-loop simulation of the pantograph-catenary interaction," Vehicle System Dynamics, vol. 51, no. 4, pp. 499-516, 2013.

[15] A. Schirrer, G. Aschauer, and S. Jakubek, "High-dynamic accurate railway catenary emulation by real-time mechanical impedance control for pantograph testing," in Simulation and Testing for Vehicle Technology, pp. 277-295, Springer, 2016.

[16] A. Schirrer, G. Aschauer, E. Talic, M. Kozek, and S. Jakubek, "Catenary emulation for hardware-in-the-loop pantograph testing with a model predictive energyconserving control algorithm," Mechatronics, vol. 41, pp. 17-28, 2017.

[17] S. Gregori, M. Tur, E. Nadal, J. Aguado, F. Fuenmayor, and F. Chinesta, "Fast simulation of the pantograph-catenary dynamic interaction," Finite Elements in Analysis and Design, vol. 129, pp. 1-13, 2017.

[18] M. Berzeri and A. A. Shabana, "Development of simple models for the elastic forces in the absolute nodal co-ordinate formulation," Journal of Sound and Vibration, vol. 235, no. 4, pp. 539-565, 2000.

[19] M. Tur, E. García, L. Baeza, and F. Fuenmayor, "A 3D absolute nodal coordinate finite element model to compute the initial configuration of a railway catenary," Engineering Structures, vol. 71, pp. 234-243, 2014.

[20] A. Collina and S. Bruni, "Numerical simulation of pantograph-overhead equipment interaction," Vehicle System Dynamics, vol. 38, no. 4, pp. 261-291, 2002.

[21] J. Ambrósio, J. Pombo, P. Antunes, and M. Pereira, "PantoCat statement of methods," Vehicle System Dynamics, vol. 53, no. 3, pp. 314-328, 2015. 
[22] J. P. Massat, C. Laurent, J. P. Bianchi, and E. Balmès, "Pantograph catenary dynamic optimisation based on advanced multibody and finite element co-simulation tools," Vehicle System Dynamics, vol. 52, no. sup1, pp. 338-354, 2014.

[23] P. Nåvik, A. Rønnquist, and S. Stichel, "Variation in predicting pantograph-catenary interaction contact forces, numerical simulations and field measurements," Vehicle System Dynamics, pp. 1-18, 2017. 